\title{
Food-web dynamics and isotopic niches in deep-sea communities residing in a submarine canyon and on the adjacent open slopes
}

Amanda W. J. Demopoulos, Jennifer McClain-Counts, Steve W. Ross, Sandra Brooke, Furu Mienis

*Corresponding author: ademopoulos@usgs.gov

Marine Ecology Progress Series 578: 19-33 (2017)

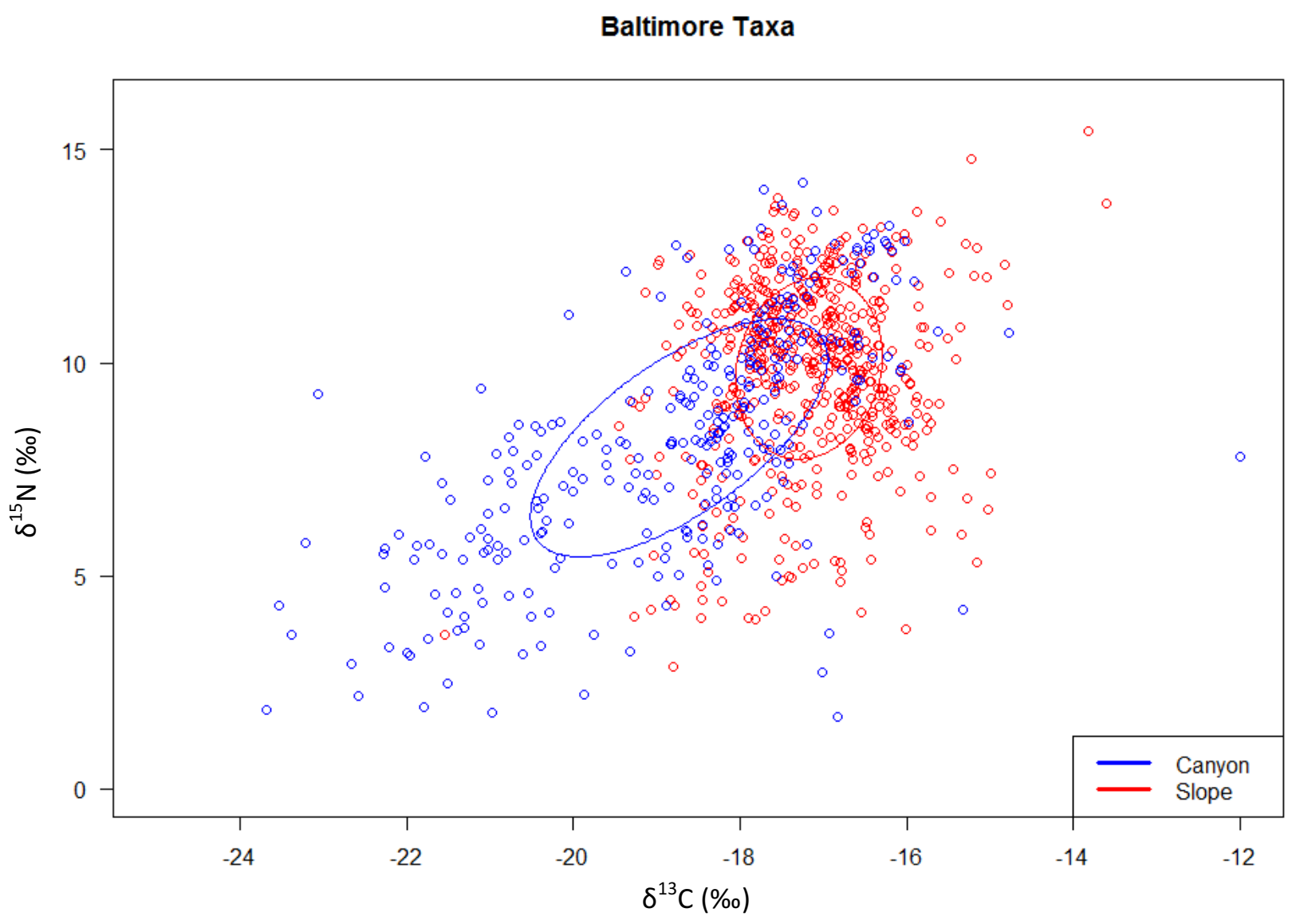

Fig. S1. Canyon and slope SEAc values (ellipses) and raw faunal isotope data. 

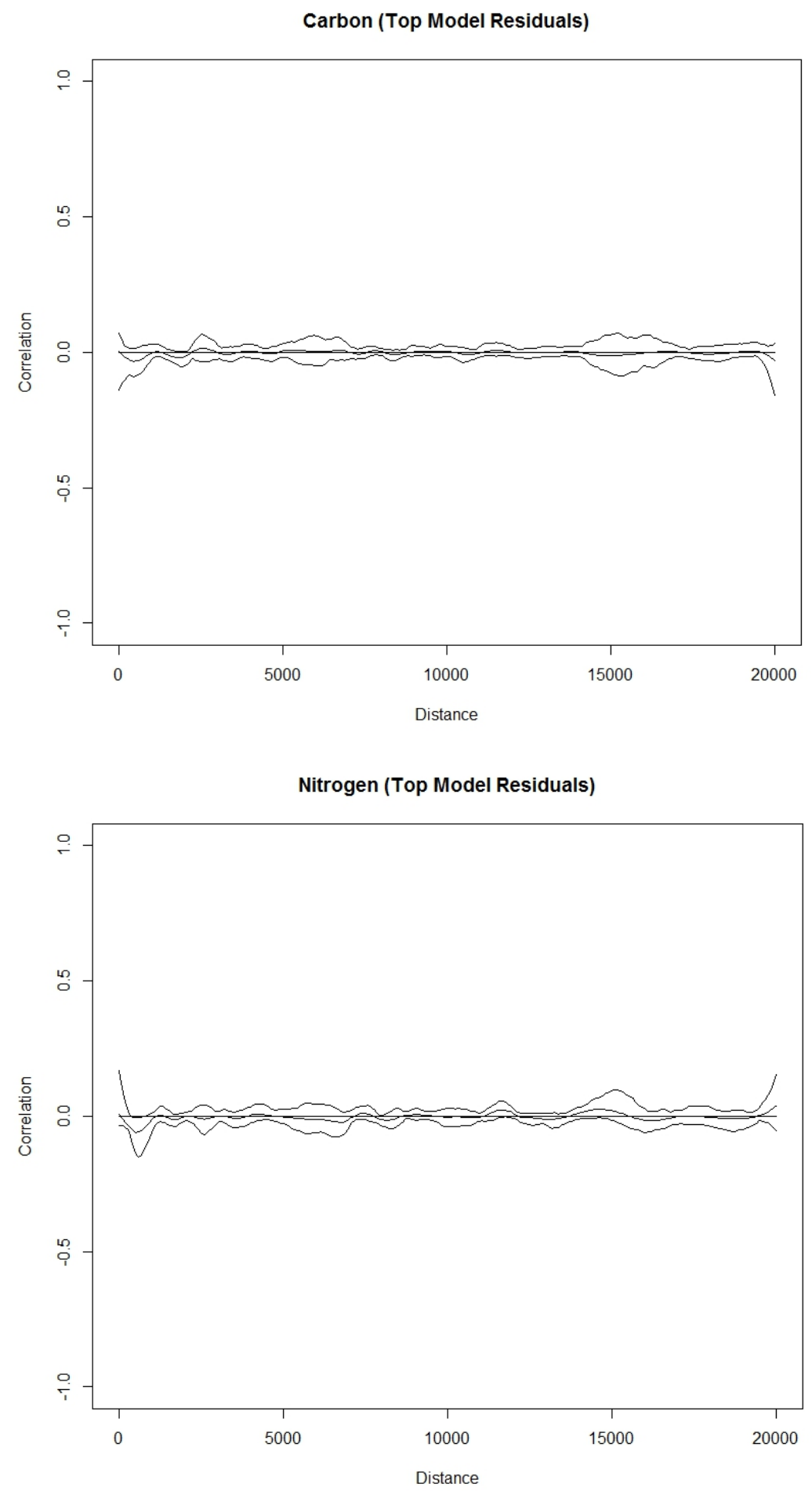

Fig. S2. Non-parametric spline correlogram showing spatial autocorrelation for the residuals from the top carbon and nitrogen models. 

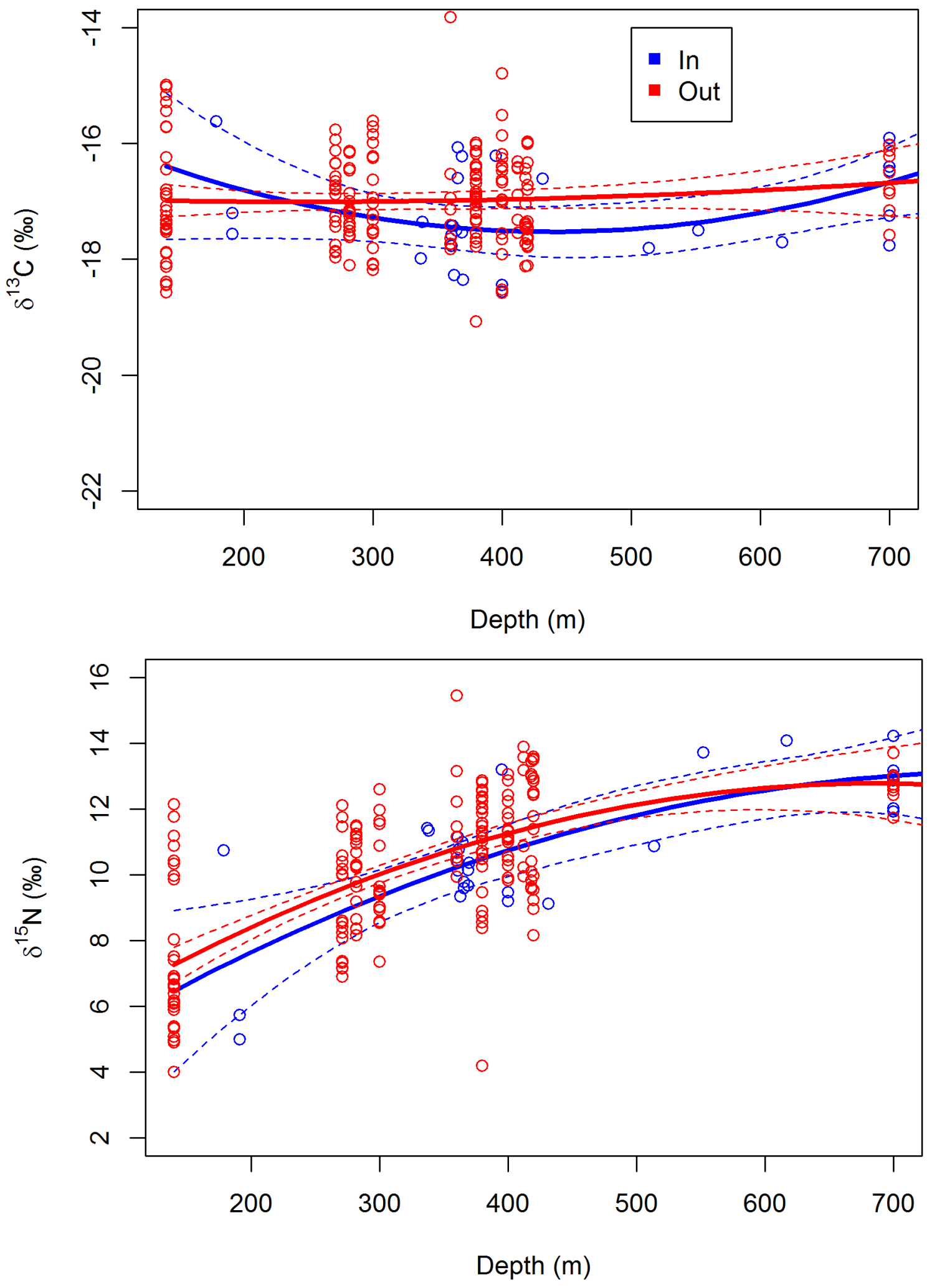

Fig. S3. OLS model predictions for $\delta^{13} \mathrm{C}$ and $\delta^{15} \mathrm{~N}$ values versus depth of benthic feeders with confidence envelopes. In $=$ canyon fauna, Out $=$ slope fauna. 


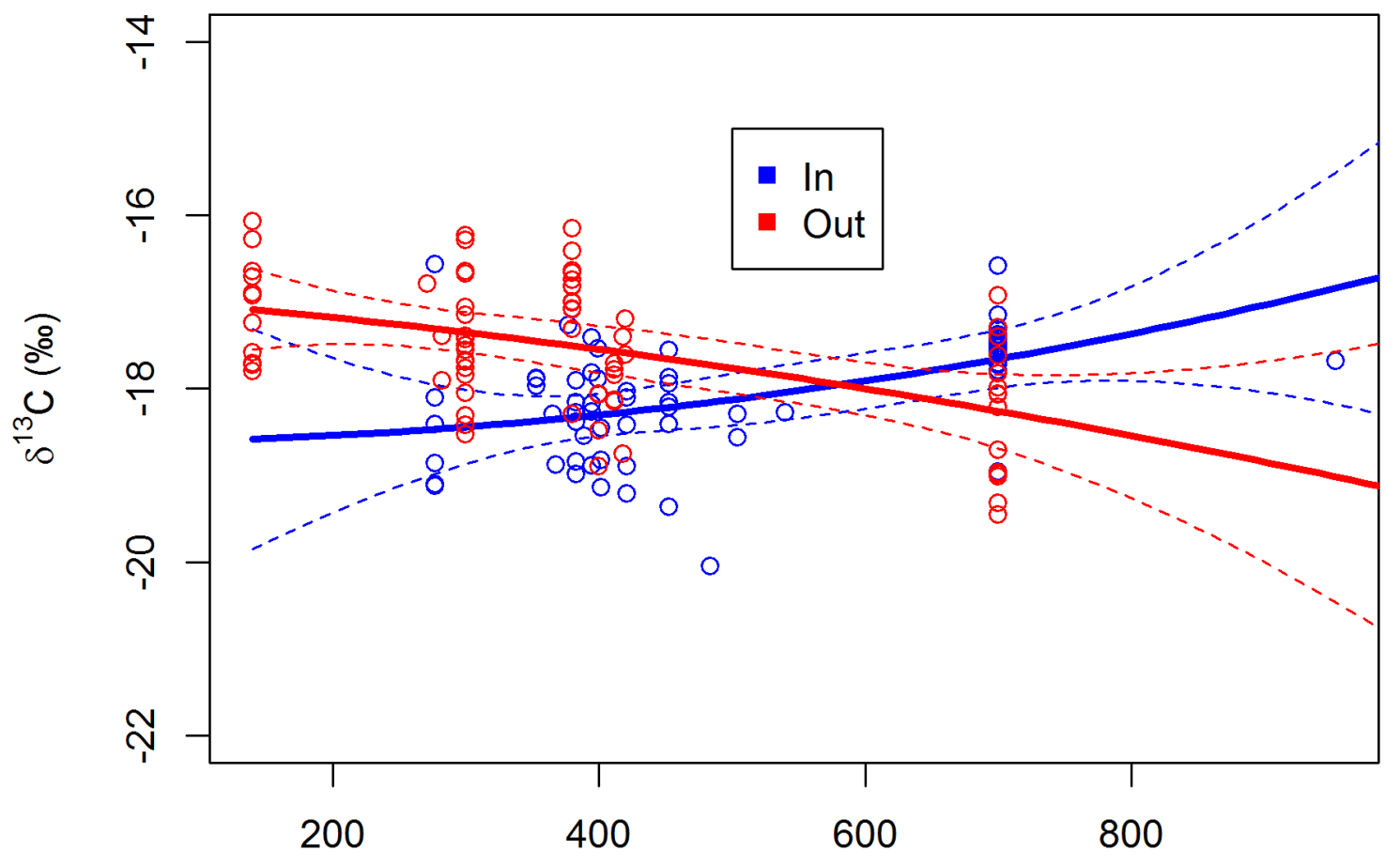

Depth $(m)$

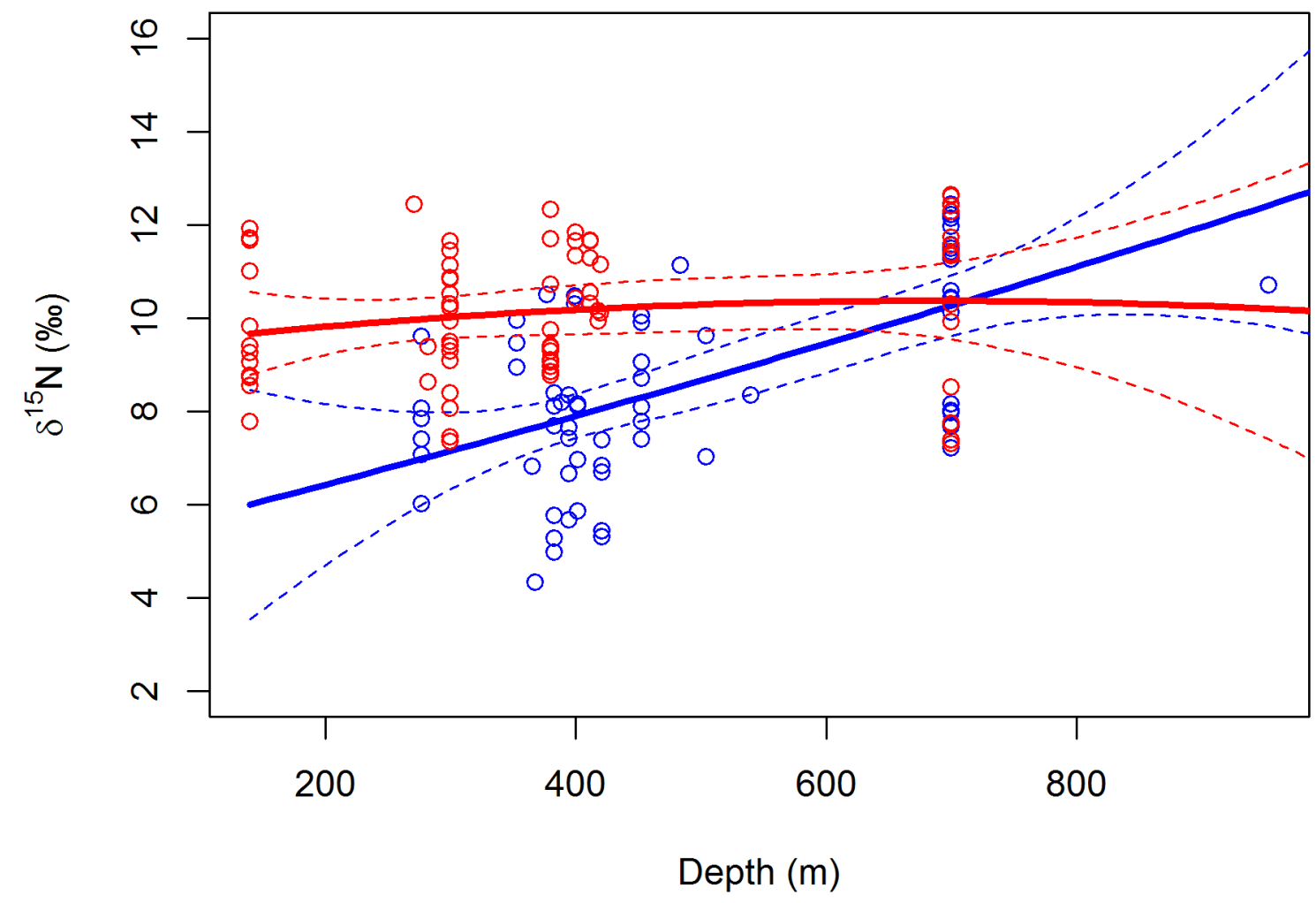

Fig. S4. OLS model predictions for $\delta^{13} \mathrm{C}$ and $\delta^{15} \mathrm{~N}$ values versus depth of pelagic feeders with confidence envelopes. $\mathrm{In}=$ canyon fauna, Out $=$ slope fauna. 


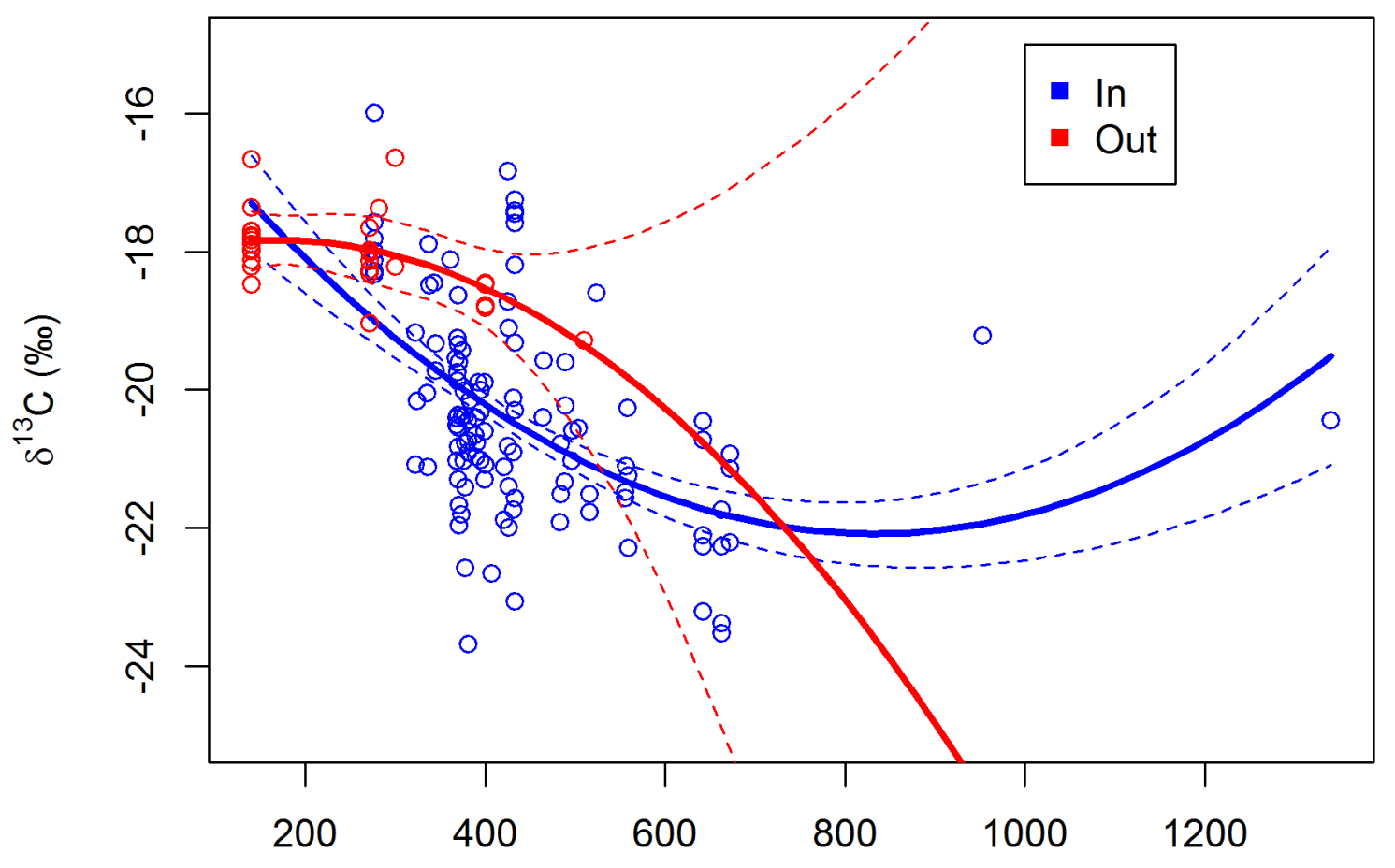

Depth $(m)$

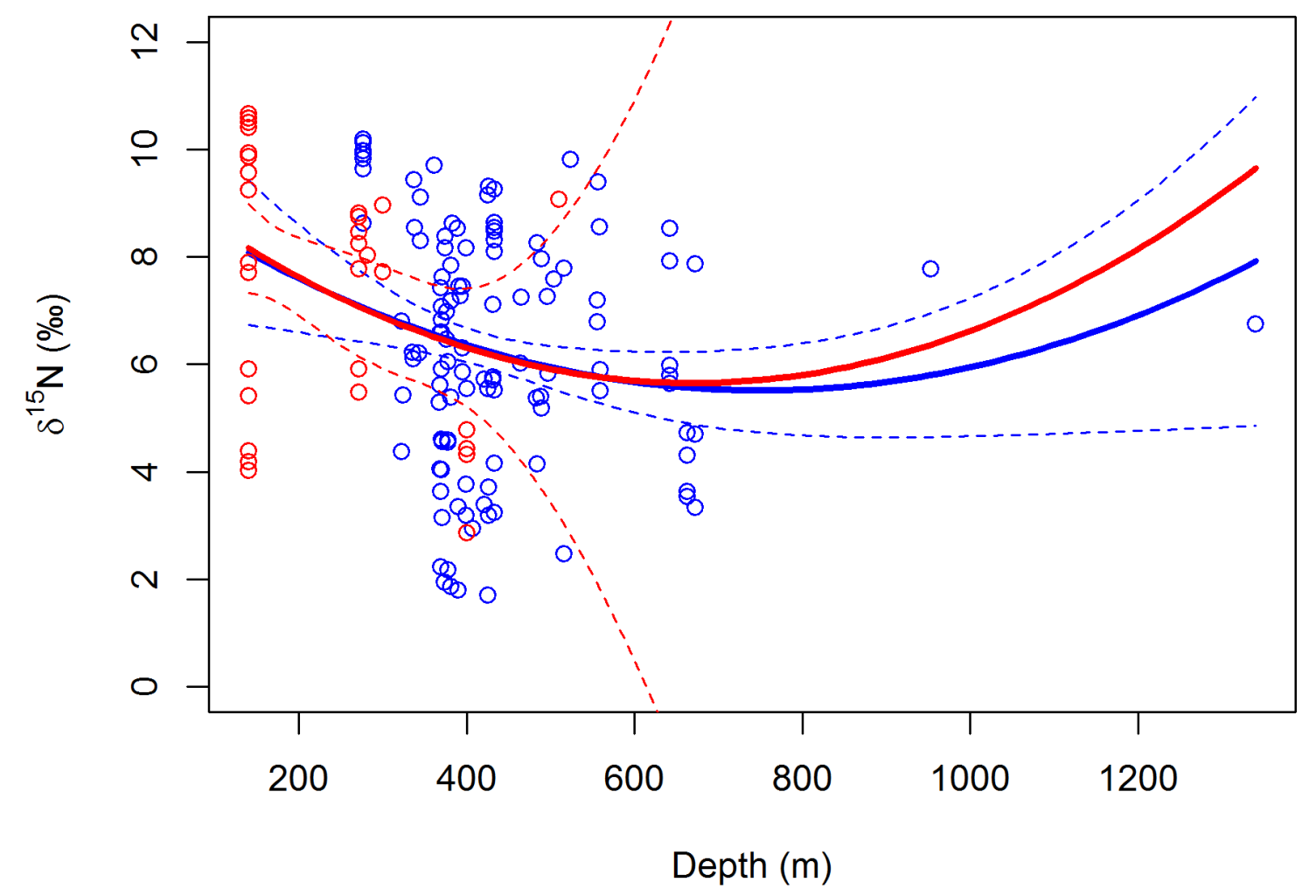

Fig. S5. OLS model predictions for $\delta^{13} \mathrm{C}$ and $\delta^{15} \mathrm{~N}$ values versus depth of suspension feeders with confidence envelopes. $\mathrm{In}=$ canyon fauna, Out $=$ slope fauna. 

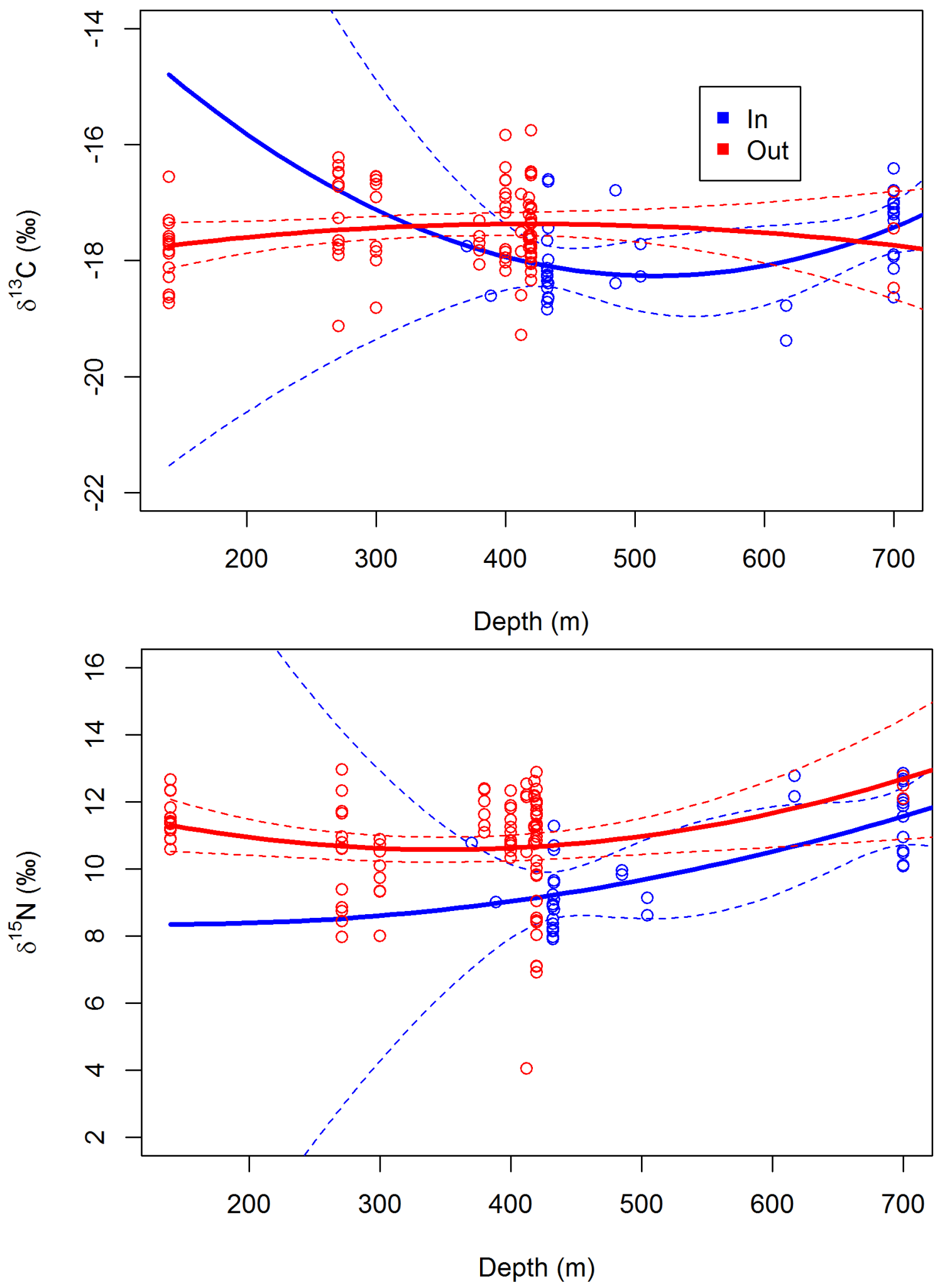

Fig. S6. OLS model predictions for $\delta^{13} \mathrm{C}$ and $\delta^{15} \mathrm{~N}$ values versus depth of suprabenthic feeders with confidence envelopes. $\mathrm{In}=$ canyon fauna, Out $=$ slope fauna. 

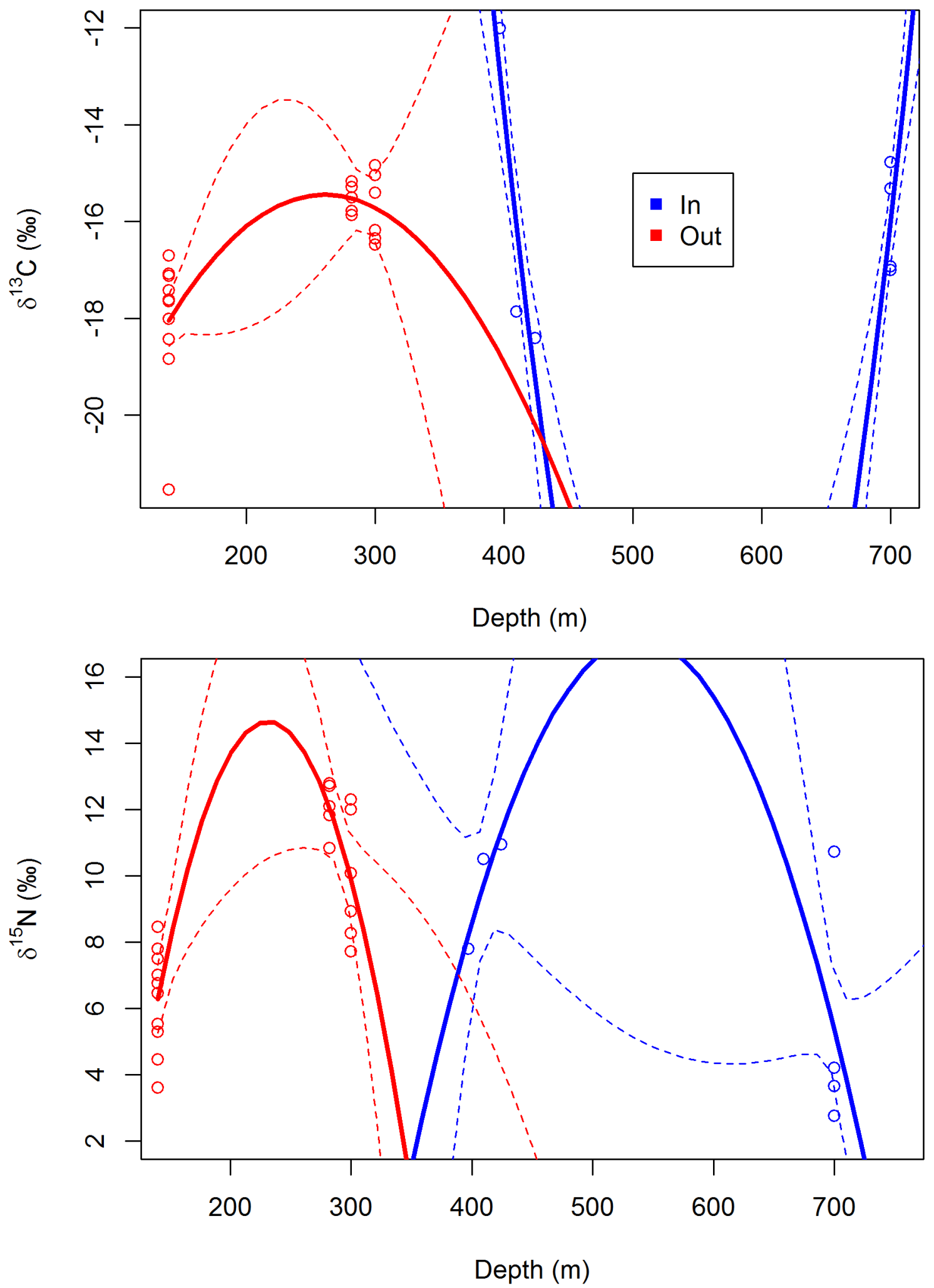

Fig. S7. OLS model predictions for $\delta^{13} \mathrm{C}$ and $\delta^{15} \mathrm{~N}$ values versus depth of deposit feeders with confidence envelopes. In $=$ canyon fauna, Out $=$ slope fauna. 

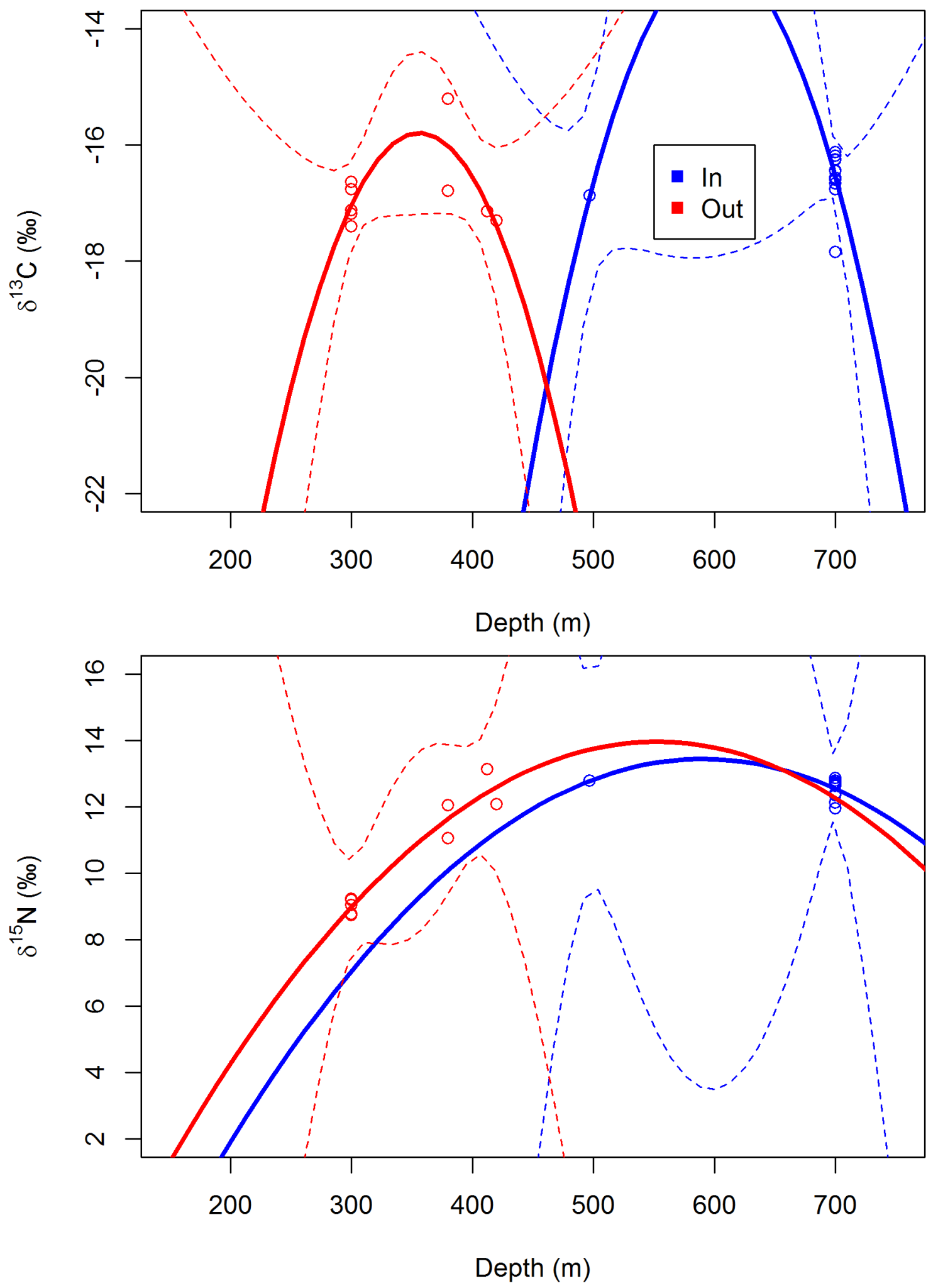

Fig. S8. OLS model predictions for $\delta^{13} \mathrm{C}$ and $\delta^{15} \mathrm{~N}$ values versus depth of epibenthic feeders with confidence envelopes. In = canyon fauna, Out $=$ slope fauna. 

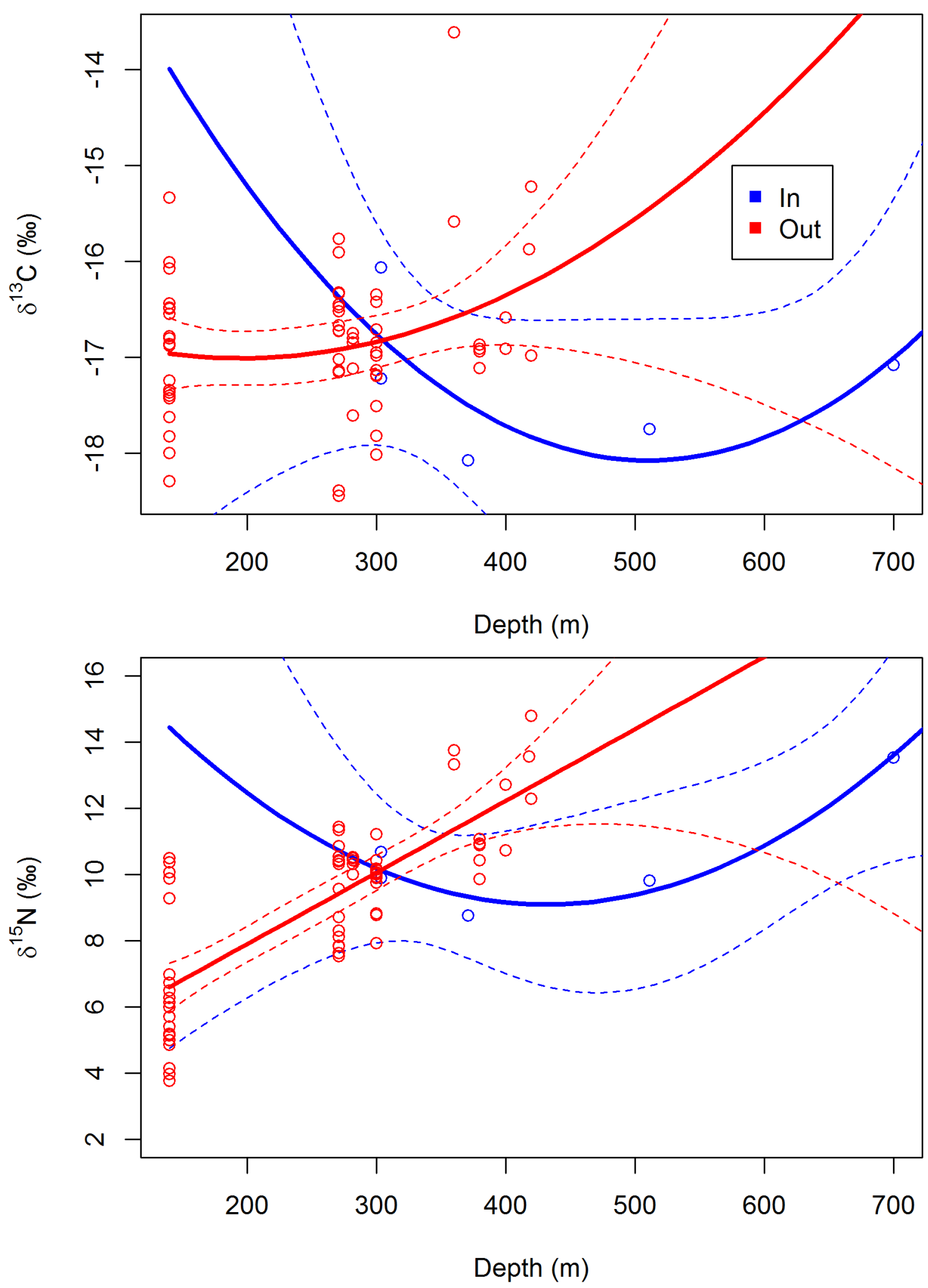

Fig. S9. OLS model predictions for $\delta^{13} \mathrm{C}$ and $\delta^{15} \mathrm{~N}$ values versus depth of infaunal feeders with confidence envelopes. $\mathrm{In}=$ canyon fauna, Out $=$ slope fauna. 


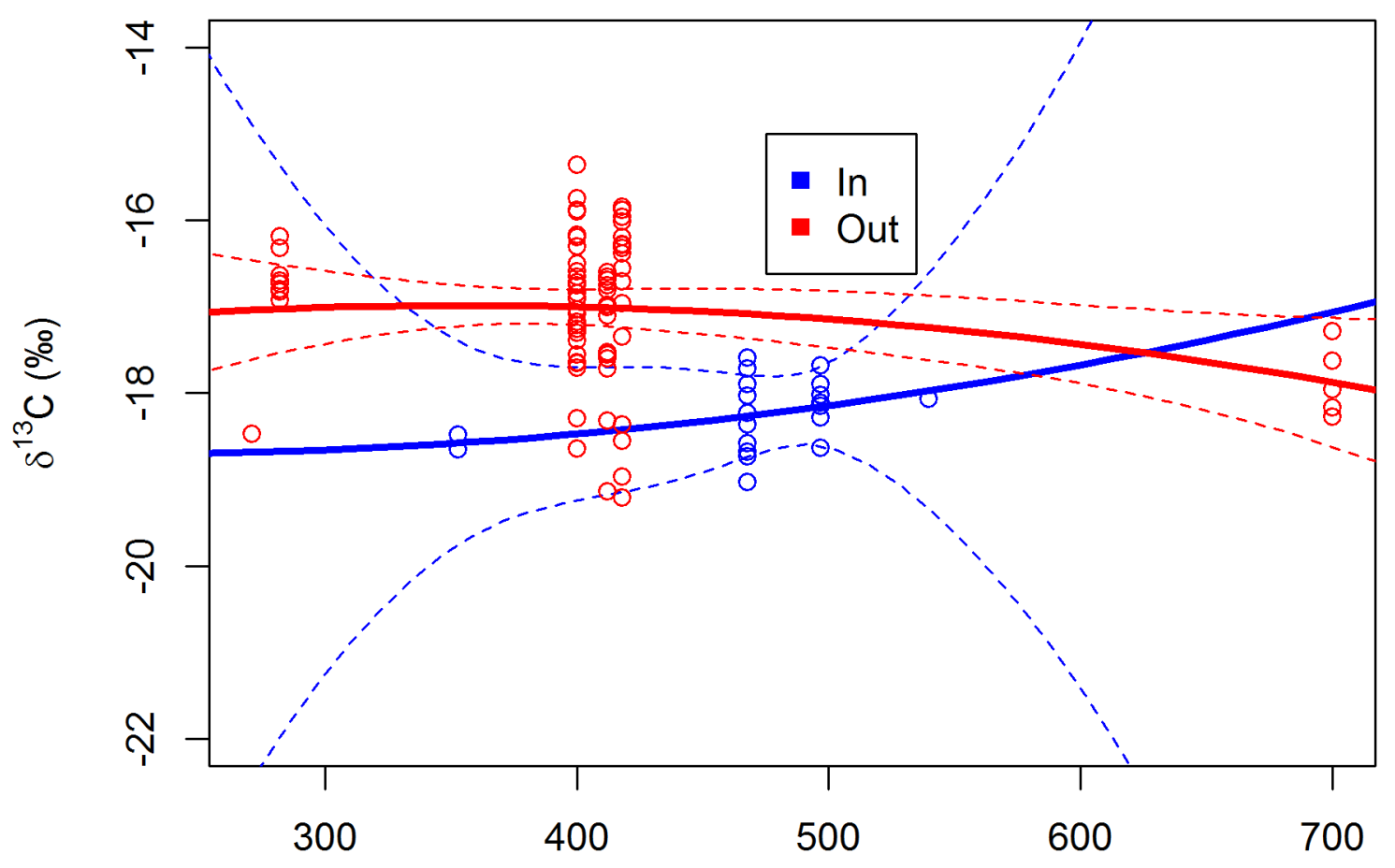

Depth $(m)$

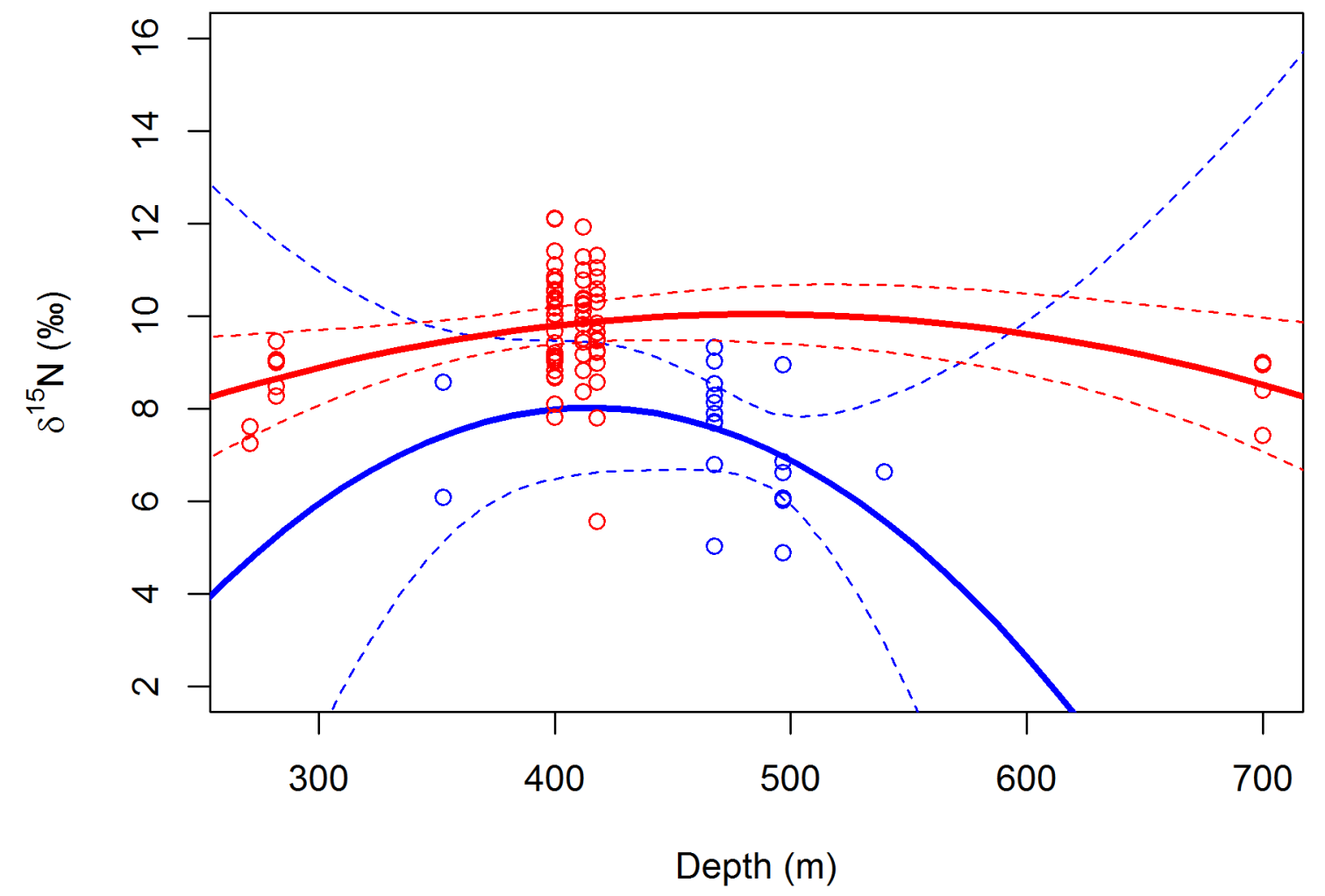

Fig. S10. OLS model predictions for $\delta^{13} \mathrm{C}$ and $\delta^{15} \mathrm{~N}$ values versus depth of unknown feeders with confidence envelopes. $\mathrm{In}=$ canyon fauna, Out $=$ slope fauna. 
Table S1. Stations where isotope samples were collected near Baltimore Canyon and the adjacent slope. OT $=$ otter trawl, $\mathrm{BC}=$ box core, $\mathrm{ROV}=$ Jason II and Kraken II. ROV depth range and location are based on bottom time. * = poor quality or gear issue. For stations using the gear CTD, BC, and Lander, the start latitude and longitude represent when gear was near/on bottom.

\begin{tabular}{|c|c|c|c|c|c|c|c|c|}
\hline Station & Date & Gear & Location & Start Latitude & Start Longitude & End Latitude & End Longitude & Depth Range (m) \\
\hline NF-2011-005 & $6 / 07 / 11$ & CTD & Canyon & 38.1777 & -73.8599 & & & $195-459$ \\
\hline NF-2011-B13 & $6 / 09 / 11$ & CTD & Canyon & 38.1580 & -73.8470 & & & $100-347$ \\
\hline NF-2011-B15 & $6 / 09 / 11$ & CTD & Canyon & 38.2063 & -73.8463 & & & $200-629$ \\
\hline NF-2012-010 & $8 / 18 / 12$ & CTD & Canyon & 38.1028 & -73.8002 & & & $0-396$ \\
\hline NF-2012-012 & $8 / 18 / 12$ & Mooring & Canyon & 38.0776 & -73.7826 & & & 1082 \\
\hline NF-2012-019 & $8 / 19 / 12$ & $\mathrm{BC}$ & Canyon & 38.2432 & -73.8436 & & & 189 \\
\hline NF-2012-024 & $8 / 20 / 12$ & CTD & Canyon & 38.1093 & -73.8252 & & & $0-854$ \\
\hline NF-2012-028 & $8 / 20 / 12$ & $\mathrm{BC}$ & Canyon & 38.2428 & -73.8435 & & & 191 \\
\hline NF-2012-029 & $8 / 20 / 12$ & $\mathrm{BC}$ & Canyon & 38.2428 & -73.8435 & & & 191 \\
\hline NF-2012-032 & $8 / 21 / 12$ & $\mathrm{BC}$ & Canyon & 38.1662 & -73.8501 & & & 563 \\
\hline NF-2012-036 & $8 / 21 / 12$ & CTD & Canyon & 38.2255 & -73.8453 & & & $0-250$ \\
\hline NF-2012-045 & $8 / 22 / 12$ & $\mathrm{BC}$ & Canyon & 38.1170 & -73.8358 & & & 840 \\
\hline NF-2012-049 & $8 / 23 / 12$ & $\mathrm{BC}$ & Canyon & 38.1175 & -73.8345 & & & 844 \\
\hline NF-2012-062 & $8 / 24 / 12$ & $\mathrm{BC}$ & Canyon & 38.0721 & -73.7733 & & & 1180 \\
\hline NF-2012-063 & $8 / 24 / 12$ & $\mathrm{BC}$ & Canyon & 38.0722 & -73.7734 & & & 1180 \\
\hline NF-2012-064 & $8 / 24 / 12$ & $\mathrm{BC}$ & Slope & 38.0625 & -73.8656 & & & 168 \\
\hline NF-2012-069 & $8 / 25 / 12$ & $\mathrm{BC}$ & Slope & 38.0626 & -73.8656 & & & 169 \\
\hline NF-2012-076 & $8 / 25 / 12$ & $\mathrm{BC}$ & Slope & 38.0435 & -73.8034 & & & 510 \\
\hline NF-2012-078 & $8 / 25 / 12$ & $\mathrm{BC}$ & Slope & 38.0434 & -73.8050 & & & 510 \\
\hline NF-2012-082 & $8 / 26 / 12$ & $\mathrm{BC}$ & Slope & 38.0138 & -73.7535 & & & 990 \\
\hline NF-2012-085 & $8 / 26 / 12$ & $\mathrm{BC}$ & Slope & 38.0139 & -73.7534 & & & 991 \\
\hline NF-2012-087 & $8 / 26 / 12$ & $\mathrm{BC}$ & Slope & 38.0139 & -73.7534 & & & 991 \\
\hline NF-2012-089 & $8 / 26 / 12$ & $\mathrm{BC}$ & Slope & 38.0112 & -73.7543 & & & 1030 \\
\hline NF-2012-093 & $8 / 27 / 12$ & $\mathrm{BC}$ & Slope & 37.9774 & -73.6694 & & & 1186 \\
\hline NF-2012-095 & $8 / 27 / 12$ & $\mathrm{BC}$ & Slope & 37.9774 & -73.6694 & & & 1186 \\
\hline NF-2012-097 & $8 / 27 / 12$ & CTD & Slope & 38.0984 & -73.9343 & & & $0-107$ \\
\hline NF-2012-100 & $8 / 27 / 12$ & CTD & Slope & 38.0580 & -73.8469 & & & $0-252$ \\
\hline NF-2012-102 & $8 / 28 / 12$ & CTD & Slope & 38.0275 & -73.7861 & & & $0-680$ \\
\hline NF-2012-105 & $8 / 28 / 12$ & CTD & Slope & 37.9823 & -73.6886 & & & $0-1066$ \\
\hline NF-2012-106 & $8 / 28 / 12$ & $\mathrm{BC}$ & Canyon & 38.2248 & -73.8445 & & & 280 \\
\hline
\end{tabular}




\begin{tabular}{|c|c|c|c|c|c|c|c|c|}
\hline Station & Date & Gear & Location & Start Latitude & Start Longitude & End Latitude & End Longitude & Depth Range (m) \\
\hline NF-2012-108 & $8 / 28 / 12$ & $\mathrm{BC}$ & Canyon & 38.2030 & -73.8494 & & & 360 \\
\hline NF-2012-110 & $8 / 29 / 12$ & $\mathrm{BC}$ & Canyon & 38.1820 & -73.8655 & & & 518 \\
\hline NF-2012-112 & $8 / 29 / 12$ & $\mathrm{BC}$ & Canyon & 38.1481 & -73.8458 & & & 652 \\
\hline NF-2012-117 & $8 / 29 / 12$ & $\mathrm{BC}$ & Canyon & 38.1290 & -73.8412 & & & 754 \\
\hline NF-2012-118 & $8 / 29 / 12$ & $\mathrm{BC}$ & Slope & 38.0724 & -73.8796 & & & 139 \\
\hline NF-2012-131 & $9 / 05 / 12$ & Lander & Canyon & 38.0424 & -73.7359 & & & 1318 \\
\hline NF-2012-132 & $9 / 06 / 12$ & Lander & Canyon & 38.1504 & -73.8492 & & & 613 \\
\hline NF-2012-133 & $9 / 06 / 12$ & OT & Slope & 38.0751 & -73.8827 & 38.0583 & -73.9001 & $136-140$ \\
\hline NF-2012-134 & $9 / 07 / 12$ & OT & Slope & 38.0427 & -73.8534 & 38.0248 & -73.8670 & $278-282$ \\
\hline NF-2012-135 & $9 / 07 / 12$ & OT & Slope & 37.9886 & -73.9031 & 37.9699 & -73.9188 & $360-380$ \\
\hline NF-2012-139 & $9 / 07 / 12$ & OT & Slope & 38.1404 & -73.7514 & 38.1564 & -73.7434 & $214-300$ \\
\hline NF-2012-140 & $9 / 08 / 12$ & OT & Slope & 38.1308 & -73.7584 & 38.1493 & -73.7462 & $290-300$ \\
\hline NF-2012-141 & $9 / 08 / 12$ & OT & Slope & 38.1426 & -73.7496 & 38.1275 & -73.7617 & $300-300$ \\
\hline NF-2012-142 & $9 / 08 / 12$ & OT & Slope & 38.1162 & -73.7548 & 38.1363 & -73.7527 & $300-360$ \\
\hline NF-2012-143 & $9 / 09 / 12$ & OT & Slope & 38.0976 & -73.7612 & 38.1091 & -73.7445 & $405-420$ \\
\hline NF-2012-144 & $9 / 09 / 12$ & OT & Slope & 38.1131 & -73.7465 & 38.0961 & -73.7559 & $400-540$ \\
\hline NF-2012-145 & $9 / 10 / 12$ & OT & Slope & 38.1051 & -73.7501 & 38.1234 & -73.7484 & $418-570$ \\
\hline NF-2012-146 & $9 / 10 / 12$ & OT & Slope & 38.0986 & -73.7593 & 38.1165 & -73.7468 & $412-422$ \\
\hline NF-2012-147 & $9 / 10 / 12$ & OT & Slope & 38.1280 & -73.7616 & 38.1129 & -73.7802 & $250-300$ \\
\hline NF-2012-148 & $9 / 11 / 12$ & CTD & Slope & 38.0275 & -73.7865 & & & $0-671$ \\
\hline NF-2012-150* & $9 / 12 / 12$ & OT & Slope & 38.0331 & -73.7677 & & & $0-700$ \\
\hline NF-2012-151 & $9 / 12 / 12$ & OT & Canyon & 38.1347 & -73.8503 & 38.1228 & -73.8432 & $700-800$ \\
\hline NF-2013-008 & $8 / 23 / 13$ & CTD & Canyon & 38.0361 & -73.7341 & & & $0-966$ \\
\hline NF-2013-009 & $8 / 24 / 13$ & CTD & Canyon & 38.0735 & -73.7855 & & & $0-966$ \\
\hline NF-2013-010 & $8 / 24 / 13$ & CTD & Canyon & 38.1487 & -73.8465 & & & $0-634$ \\
\hline NF-2013-011 & $8 / 24 / 13$ & CTD & Slope & 38.0490 & -73.8301 & & & $0-335$ \\
\hline NF-2013-015 & $8 / 24 / 13$ & CTD & Slope & 38.0490 & -73.8188 & & & $0-440$ \\
\hline NF-2013-017 & $8 / 24 / 13$ & CTD & Slope & 38.0489 & -73.8132 & & & $0-508$ \\
\hline NF-2013-018 & $8 / 24 / 13$ & CTD & Canyon & 38.0908 & -73.8062 & & & $0-905$ \\
\hline NF-2013-019 & $8 / 24 / 13$ & CTD & Canyon & 38.1060 & -73.8232 & & & $0-896$ \\
\hline NF-2013-020 & $8 / 25 / 13$ & CTD & Canyon & 38.1241 & -73.8378 & & & $0-773$ \\
\hline NF-2013-021 & $8 / 25 / 13$ & CTD & Canyon & 38.1469 & -73.8455 & & & $0-632$ \\
\hline RB-2013-079 & $5 / 16 / 13$ & $\mathrm{BC}$ & Canyon & 38.0614 & -73.6063 & & & 1608 \\
\hline
\end{tabular}




\begin{tabular}{|c|c|c|c|c|c|c|c|c|}
\hline Station & Date & Gear & Location & Start Latitude & Start Longitude & End Latitude & End Longitude & Depth Range (m) \\
\hline RB-2013-080 & $5 / 17 / 13$ & $\mathrm{BC}$ & Canyon & 38.0614 & -73.6063 & & & 1606 \\
\hline RB-2013-081 & $5 / 17 / 13$ & $\mathrm{BC}$ & Canyon & 38.0613 & -73.6054 & & & 1608 \\
\hline ROV-2012-NF-01 & $8 / 18 / 12$ & ROV & Canyon & 38.1471 & -73.8434 & 38.1491 & -73.8380 & $450-634$ \\
\hline ROV-2012-NF-02 & $8 / 19 / 12$ & $\mathrm{ROV}$ & Canyon & 38.1484 & -73.8389 & 38.1448 & -73.8339 & $402-530$ \\
\hline ROV-2012-NF-03 & $8 / 21 / 12$ & ROV & Canyon & 38.1071 & -73.8085 & 38.1267 & -73.8031 & $303-827$ \\
\hline ROV-2012-NF-04 & $8 / 22 / 12$ & ROV & Canyon & 38.0856 & -73.7844 & 38.1028 & -73.7839 & $537-1001$ \\
\hline ROV-2012-NF-05 & $8 / 23 / 12$ & ROV & Canyon & 38.1379 & -73.8359 & 38.1374 & -73.8333 & $400-540$ \\
\hline ROV-2012-NF-06 & $8 / 24 / 12$ & ROV & Canyon & 38.1396 & -73.8357 & 38.1470 & -73.8328 & $234-530$ \\
\hline ROV-2012-NF-09 & $8 / 28 / 12$ & ROV & Canyon & 38.1522 & -73.8416 & 38.1529 & -73.8337 & $313-574$ \\
\hline ROV-2012-NF-10 & $8 / 29 / 12$ & ROV & Canyon & 38.1685 & -73.8524 & 38.1613 & -73.8588 & $425-574$ \\
\hline ROV-2012-NF-11 & $8 / 30 / 12$ & ROV & Canyon & 38.0931 & -73.8066 & 38.0885 & -73.8303 & $446-938$ \\
\hline ROV-2012-NF-13 & $9 / 06 / 12$ & ROV & Canyon & 38.1595 & -73.8557 & 38.1566 & -73.8651 & $404-478$ \\
\hline ROV-2012-NF-16 & $9 / 09 / 12$ & ROV & Canyon & 38.1771 & -73.8611 & 38.1892 & -73.8563 & $343-551$ \\
\hline ROV-2012-NF-17 & $9 / 10 / 12$ & ROV & Canyon & 38.1170 & -73.8413 & 38.1186 & -73.8478 & $569-830$ \\
\hline ROV-2012-NF-18 & $9 / 11 / 12$ & ROV & Canyon & 38.1170 & -73.8456 & 38.1155 & -73.8500 & $521-748$ \\
\hline ROV-2012-NF-19 & $9 / 12 / 12$ & ROV & Canyon & 38.1553 & -73.8406 & 38.1508 & -73.8353 & $302-608$ \\
\hline ROV-2013-RB-690 & $5 / 17 / 13$ & ROV & Canyon & 38.1705 & -73.8377 & 38.1608 & -73.8332 & $289-464$ \\
\hline
\end{tabular}


Table S2. Feeding group assignments by taxa and corresponding literature for fauna collected from Baltimore Canyon and adjacent slope. NA refers to taxa with an unknown feeding group assignment and/or no literature. Species marked with $\mathrm{a}^{\wedge}$ were lacking published feeding group assignments, so feeding group assignment was based on published data from a similar species (family or genus level).

\begin{tabular}{|c|c|c|}
\hline Species ID & Feeding Group & References \\
\hline \multicolumn{3}{|l|}{ SOURCES } \\
\hline POM & Producer & NA \\
\hline Sediment & Sediment & NA \\
\hline \multicolumn{3}{|l|}{ ANNELIDA } \\
\hline Annelida & Unknown & NA \\
\hline Polychaeta & Unknown & NA \\
\hline Hyalinoecia sp. & Deposit & 1 \\
\hline Onuphidae & Deposit & 1 \\
\hline Polynoidae & Infaunal & 1,2 \\
\hline \multicolumn{3}{|l|}{ ARTHROPODA } \\
\hline Amphipoda & Benthic/Pelagic & \\
\hline Unciola sp. & Deposit & $73,74,75,76,77$ \\
\hline Themisto sp. $^{\wedge}$ & Pelagic & 13 \\
\hline Aristeus antillensis & Benthic & 3 \\
\hline Cancer plebejus & Benthic & 5 \\
\hline Eumunida picta & Benthic & 6 \\
\hline Eumunida sp. & Benthic & 6 \\
\hline Paguristes lymani ${ }^{\wedge}$ & Benthic & 7 \\
\hline Chaceon quinquedens & Suprabenthic & 11,12 \\
\hline cf. Chaceon sp. & Suprabenthic & 11,12 \\
\hline Latreillia elegans ${ }^{\wedge}$ & Benthic & 14 \\
\hline Agononida longipes $^{\wedge}$ & Benthic & $3,16,17$ \\
\hline Munida iris ${ }^{\wedge}$ & Benthic & 3 \\
\hline Munida valida ${ }^{\wedge}$ & Benthic & 3 \\
\hline Homarus americanus & Epibenthic & 11,12 \\
\hline Atlantopandalus propinqvus & Suprabenthic & 18 \\
\hline Dichelopandalus leptocerus $^{\wedge}$ & Pelagic & 19 \\
\hline Dichelopandalus sp.^ & Pelagic & 19 \\
\hline Pandalus borealis ${ }^{\wedge}$ & Suprabenthic & 18 \\
\hline Parapenaeus politus $^{\wedge}$ & Infaunal & 20 \\
\hline Parapenaeus sp.^ & Infaunal & 20 \\
\hline Rochinia crassa $a^{\wedge}$ & Suprabenthic & 21 \\
\hline Bathynectes maravigna & Epibenthic & 22 \\
\hline Processa guyanae & Pelagic & 23 \\
\hline Acetes americanus carolinae & Pelagic & 19 \\
\hline Mesopenaeus tropicalis & Benthic & 78 \\
\hline Brachyura sp. & Unknown & NA \\
\hline Shrimp spp. & Unknown & NA \\
\hline Bentheuphausia amblyops & Pelagic & 4 \\
\hline Meganyctiphanes norvegica & Pelagic & 8 \\
\hline Nyctiphanes couchii ${ }^{\wedge}$ & Pelagic & 9,10 \\
\hline Thysanoessa macrura ${ }^{\wedge}$ & Pelagic & 8 \\
\hline
\end{tabular}


CHORDATA - INVERT

Salpida

\section{CHORDATA - FISH}

Myxine glutinosa

Scyliorhinus retifer

Leucoraja garmani

Nemichthys curvirostris $\wedge$

Nemichthys scolopaceus

Synaphobranchus affinis ${ }^{\wedge}$

Synaphobranchus kaupii

Argentina striata ${ }^{\wedge}$

Chlorophthalmus agassizi

Parasudis truculenta

Arctozenus risso

Enchelyopus cimbrius

Coelorinchus caelorhincus

Malacocephalus occidentalis

Nezumia bairdii

Macrouridae sp.

Merluccius albidus

Phycis chesteri

Urophycis chuss

Urophycis regia

Chaunax suttkusi ${ }^{\wedge}$

Lophius americanus

Dibranchus atlanticus

Notacanthus chemnitzii

Hoplostethus mediterraneus

Diaphus sp.

Myctophidae

Lepophidium profundorum

Phosichthyidae sp.

Polymetme thaeocoryla

Argyropelecus aculeatus

Maurolicus weitzmani

Polyipnus asteroids $^{\wedge}$

Stomias boa ferox

Peristedion miniatum

Peristedion truncatum $^{\wedge}$

Helicolenus dactylopterus

Monolene sessilicauda ${ }^{\wedge}$

Symphurus billykrietei ${ }^{\wedge}$

Symphurus nebulosus ${ }^{\wedge}$

Symphurus stigmosus ${ }^{\wedge}$

Citharichthys arctifrons

Hipploglossina oblonga

Glyptocephalus cynoglossus
Suspension

61

Benthic

$41,62,71$

Benthic

Benthic

41,63

63

Pelagic

63

Pelagic

Pelagic

63

$35,44,45$

Pelagic

Pelagic

$34,35,36,44,45,64$

41

Benthic 41,63

Pelagic 67

Pelagic 63

Epibenthic 26,27,64

Benthic

Suprabenthic

$29,30,31,32,33,64$

65

Suprabenthic

$31,34,35,64$

Benthic

64

Suprabenthic

$28,36,37$

Suprabenthic

Suprabenthic

$24,36,37$

41

Suprabenthic

Suprabenthic

$24,36,37,64$

67

Benthic

$24,25,26,64$

Infaunal

35,38

Infaunal

67

63

66

66

41,63

66

63

63,66

41,63

67

63

41,63

41,63

Benthic

$42,43,64$

Benthic

69,70

68,72

Infaunal

68,72

Infaunal

68,72

Infaunal

39,40

Infaunal

41,63

Benthic

$24,28,29,32,40,41$ 
Lycenchelys verrillii

Lycodes terraenovae ${ }^{\wedge}$

CNIDARIA

Actiniaria sp. ${ }^{\wedge}$

Actiniaria sp. $3^{\wedge}$

Actinoscyphia sp.

Bolocera sp.

Halcurias sp.

Hormathiidae sp.*

Alcyonacea sp. $1^{\wedge}$

Alcyonacea sp. $2^{\wedge}$

Anthothela grandiflora

Paragorgia arborea

Paramuricea placomus ${ }^{\wedge}$

Primnoa resedaeformis

Pennatulacea sp.^

Dasmosmilia lymani

Desmophyllum dianthus

Lophelia pertusa

Zoantharia sp.

Hydrozoa

\section{ECHINODERMATA}

Asteroidea $^{\wedge}$

Sclerasterias contorta ${ }^{\wedge}$

Astropecten alligator ${ }^{\wedge}$

Persephonaster echinulatus

Poraniidae

Cidaris sp.

Echinus tylodes

Ophiuroidea sp.

Amphiura otteri $^{\wedge}$

\section{MOLLUSCA}

Bathyteuthis sp.^

Brachioteuthis beani ${ }^{\wedge}$

Illex cf. illecebrosus

cf. Semirossia tenera ${ }^{\wedge}$

Rossia megaptera ${ }^{\wedge}$

Semirossia tenera ${ }^{\wedge}$

Gastropoda

Colus stimpsoni

\section{PORIFERA}

Porifera sp.

Cladorhizidae
Epibenthic

Epibenthic

$34,35,64$

36

Suspension

46,47

Suspension

46

Suspension

47

Suspension

47

Suspension

47

Suspension

51

Suspension

48

Suspension

48

Suspension

50

Suspension

50

Suspension

52

Suspension

50

Suspension

48

Suspension

50

Suspension

50

Suspension

49,50

Suspension

50

Suspension

49

Benthic 53

Benthic 53

Infaunal 53

Benthic $\quad 53,54$

Deposit 53

Deposit 53

Deposit 53

Deposit/Suspension 53

Deposit/Suspension 2,53

$\begin{array}{ll}\text { Benthic/Pelagic } & 55 \\ \text { Benthic/Pelagic } & 55 \\ \text { Pelagic } & 55 \\ \text { Benthic/Pelagic } & 55 \\ \text { Benthic } & 55 \\ \text { Benthic/Pelagic } & 55 \\ \text { Unknown } & \text { NA } \\ \text { Deposit } & 56\end{array}$

Suspension $\quad 59$

Suspension

60

*1. Fauchald and Jumars 1979, 2. Fanelli et al. 2011, 3. Cartes 1998, 4. Mauchline and Fisher 1969, 5. Drummond-Davis et al. 1982, 6. Lovrich and Thiel 2011, 7. Schembri 1982, 8. Sameoto 1980, 9. Dalley and McClatchie 1989, 10. Ritz et al. 1990, 11. Farlow 1980, 12. Gerrior 1981, 13.

Pakhomov and Perissinotto 1996, 14. Castro et al. 2003, 15. Bowman and Gruner 1973, 16. Hudson 
et al. 2003, 17. Romero et al. 2004, 18. Hopkins et al. 1993, 19. Hopkins et al. 1994, 20. Kapiris 2004, 21. Carlier et al. 2009, 22. Cartes et al. 2002, 23. Cartes et al. 2010, 24. Maurer and Bowman 1975, 25. Armstrong, et al. 1996, 26. Johnson et al. 2008, 27. Keats and Steele 1990, 28. Langton and Bowman 1980, 29. Bowmen and Michaels 1984, 30. Blaber and Bulman 1987, 31. RodriguezMarin et al. 1994, 32. Román et al. 2004, 33. Madurell and Cartes 2006, 34. Houston and Haedrich 1986, 35. Crabtree et al. 1991, 36. Sedberry and Musick 1978 , 37. Garrison and Link 2000, 38.

Scott and Scott 1988, 39. Langton and Bowman 1981, 40. Link 2002, 41. Bowman et al. 2000, 42. Consoli et al. 2010, 43. Neves, et al. 2012, 44. Robins 1968, 45. Merret and Marshall 1980, 46. Chintiroglou and Koukouras 1992, 47. Ammons and Daly 2008, 48. Lewis 1982, 49. Duineveld et al. 2007, 50. Freiwald and Roberts 2005, 51. Mincks et al. 2008, 52. Coma et al. 2001, 53. Jangoux and Lawrence 1982, 54. Pequegnat et al. 1983, 55. Hoving et al. 2014, 56. Taylor and Taylor 1977, 57. Brooks et al. 1987, 58. MacAvoy, et al. 2002, 59. Gage and Tyler 1991, 60. Vacelet and BouryEsnault 1995, 61. Madin 1974, 62. Auster 2005, 63. Collette and Klein-MacPhee 2002, 64. Horton 2015, 65. Macpherson and Roel 1987, 66. McClain-Counts 2010, 67. McEachran and Frechhelm 1998, 68. Munroe 1990, 69. Powell and Schwartz 1979, 70. Schintu et al. 1994, 71. Shelton 1978, 72. Stickney 1976, 73. Schaffner and Boesch 1982, 74. Biernbaum and Wenner 1993, 75.

Dickinson et al. 1980, 76. Wilson et al. 2009, 77. Wildish and Dadswell 1985, 78. Soto et al. 2000 
Table S3. Stable isotope values $\left(\delta^{13} \mathrm{C}\right.$ and $\left.\delta^{15} \mathrm{~N}\right)$ for POM, surface sediments $(0-2 \mathrm{~cm})$, sediment trap samples, invertebrates and fishes collected from Baltimore Canyon and the adjacent slopes. Where $\mathrm{FG}$ is the assigned feeding group $(\mathrm{IN}=$ infaunal, $\mathrm{EB}=$ epibenthic, $\mathrm{SB}=$ suprabenthic, $\mathrm{BE}=$ benthic, $\mathrm{BP}=$ benthic/pelagic, $\mathrm{PE}=$ pelagic, $\mathrm{DS}=$ deposit/suspension, $\mathrm{SS}=$ suspension, and $\mathrm{UK}=$ unknown, $\mathrm{PR}=$ producer/detritus, $\mathrm{SE}=$ sediment). $\mathrm{N}$ is the number of specimens analyzed and average \%o values ( \pm S.E.), C:N is the ratio of carbon to nitrogen and TL is the calculated trophic level $( \pm$ propagated error, see Methods). C:N data for POM were calculated from actual amount of material analyzed but was not available for all filters. The following $\mathrm{N}$ values represent number of samples used in $\mathrm{C}: \mathrm{N}$ calculations for POM: $\mathrm{n}=2$ for BC POM in (bottom), $n=2$ for BC POM in (surface), $n$ $=3$ for BC POM out (bottom), $\mathrm{n}=3$ for BC POM out (surface). For additional information regarding FG classification, refer to Table S2.

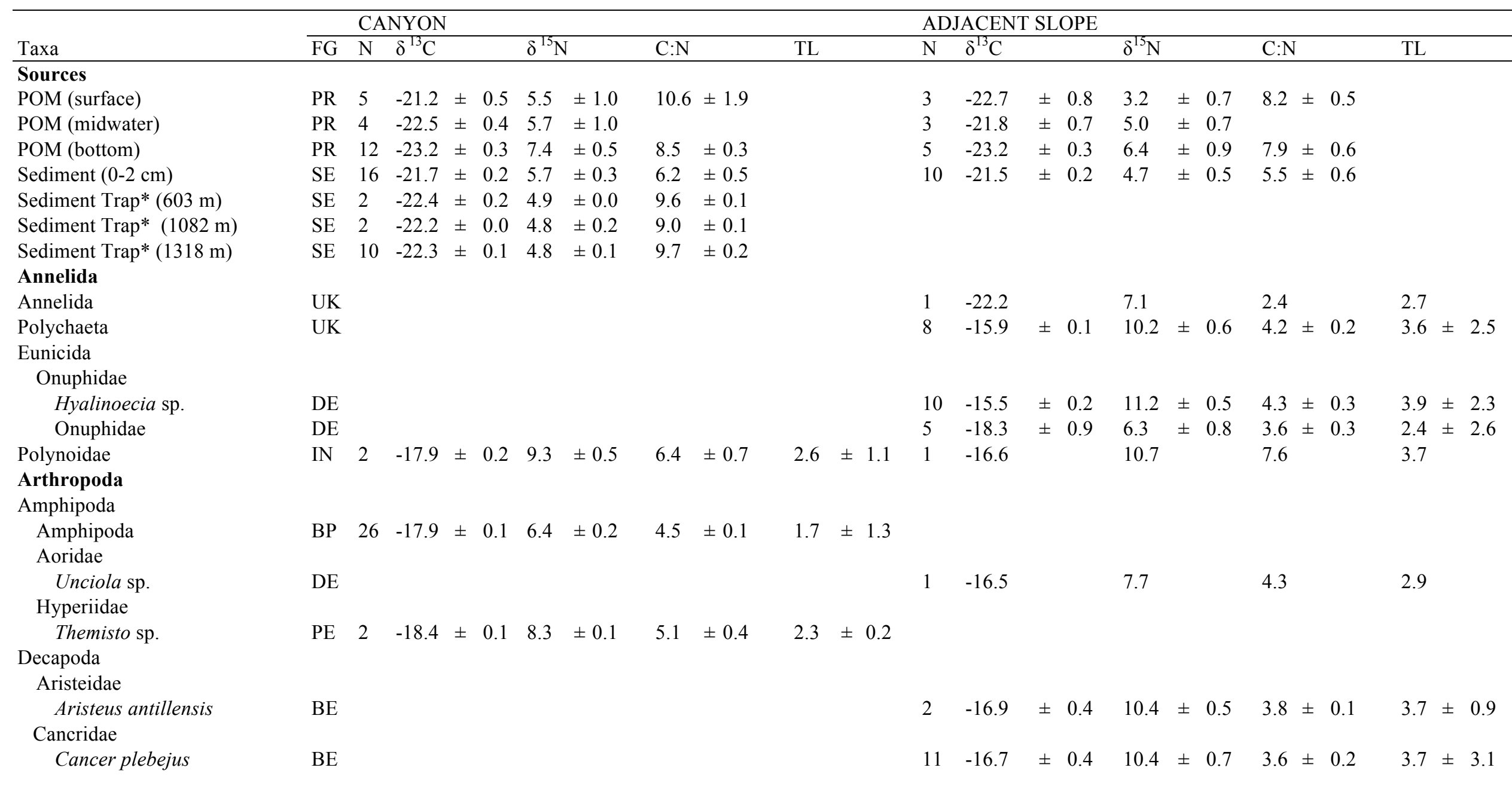




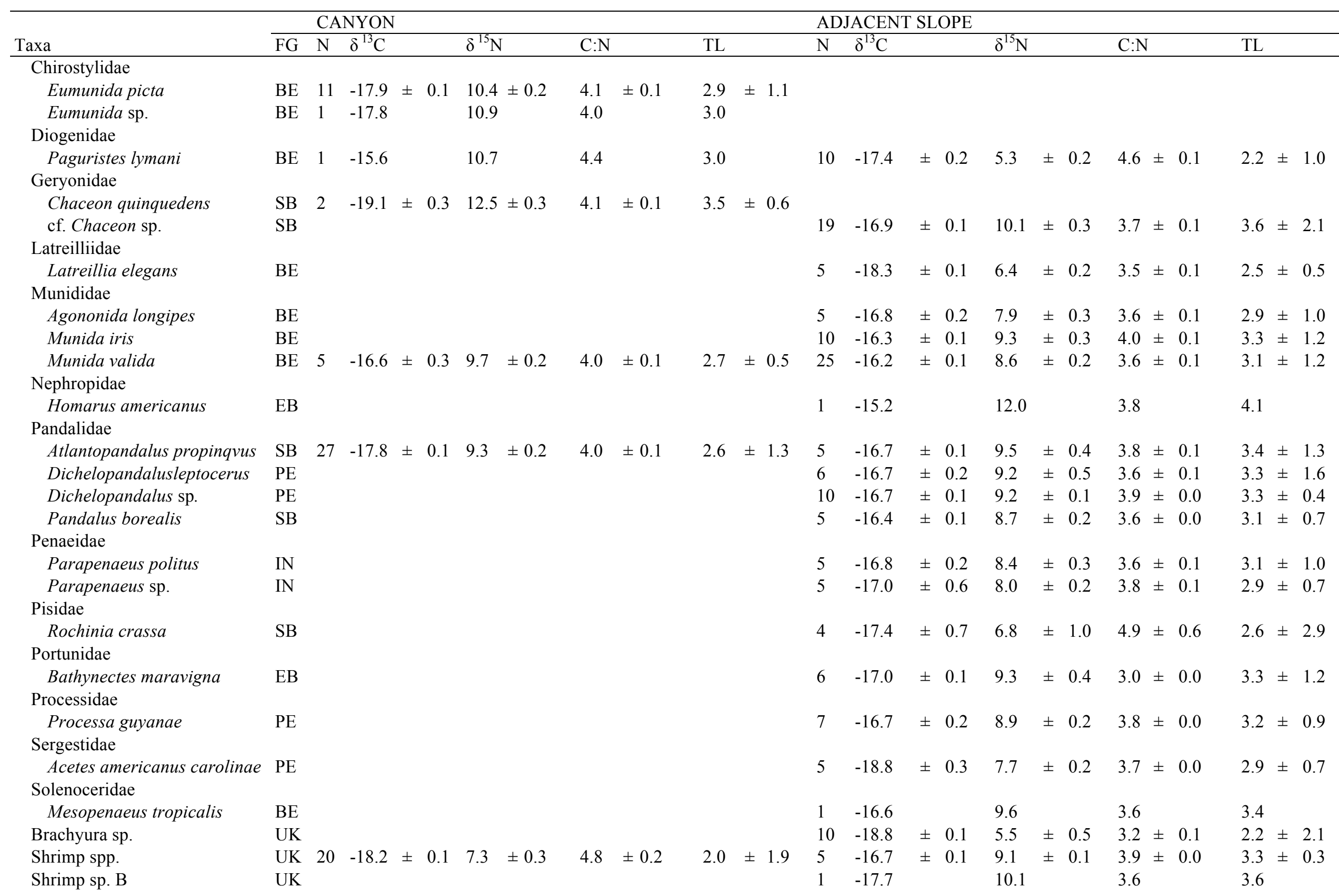




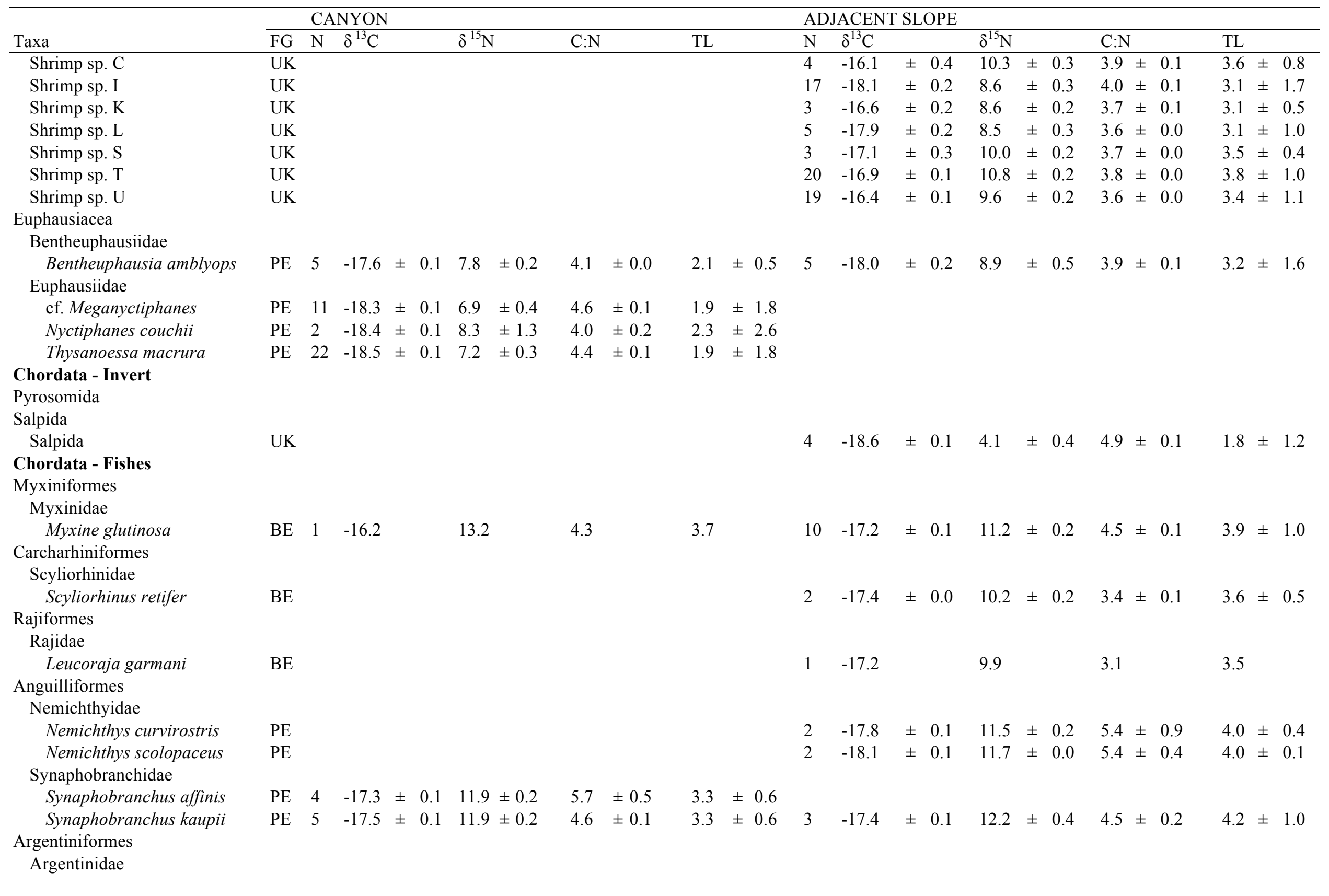




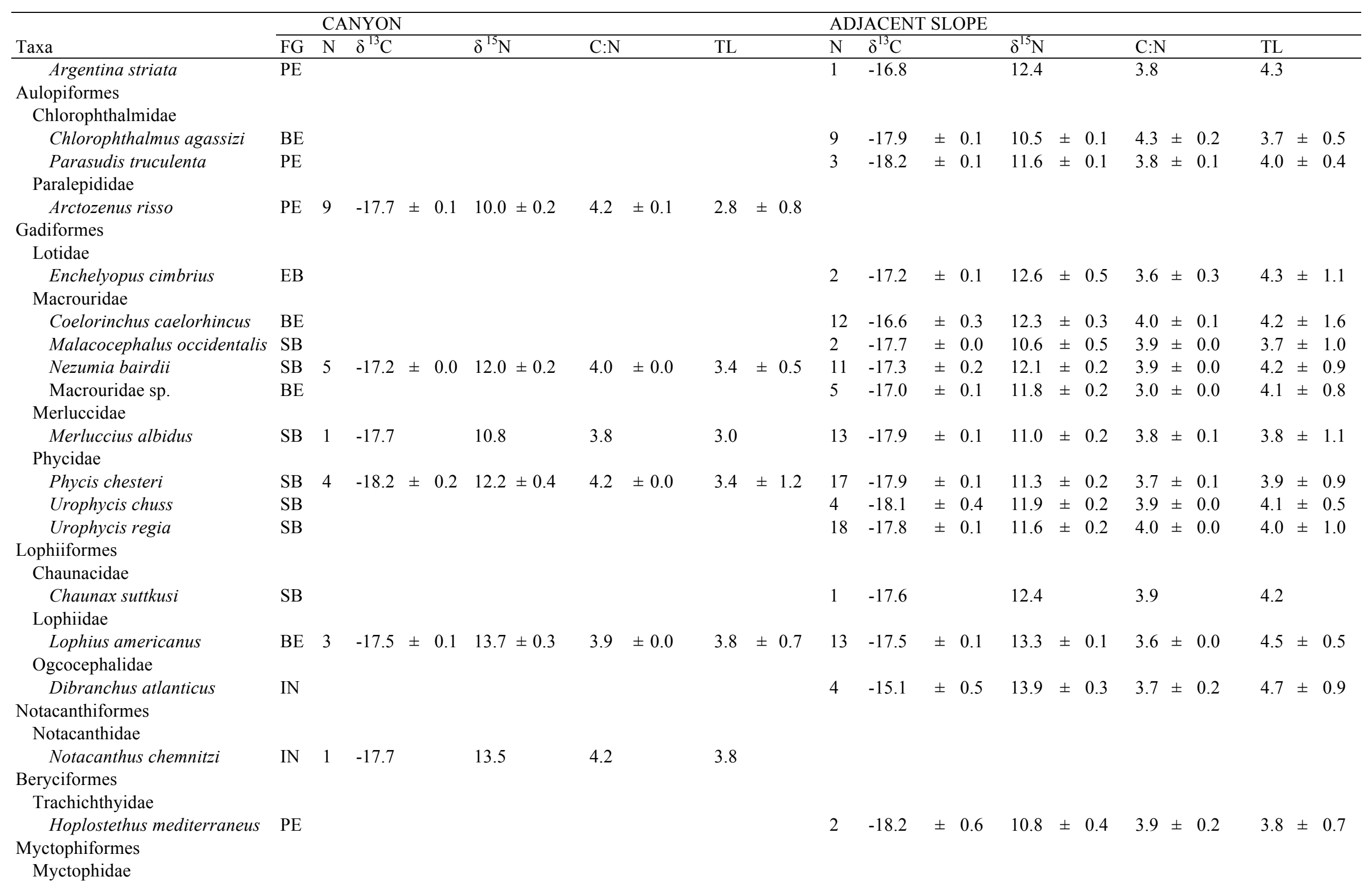




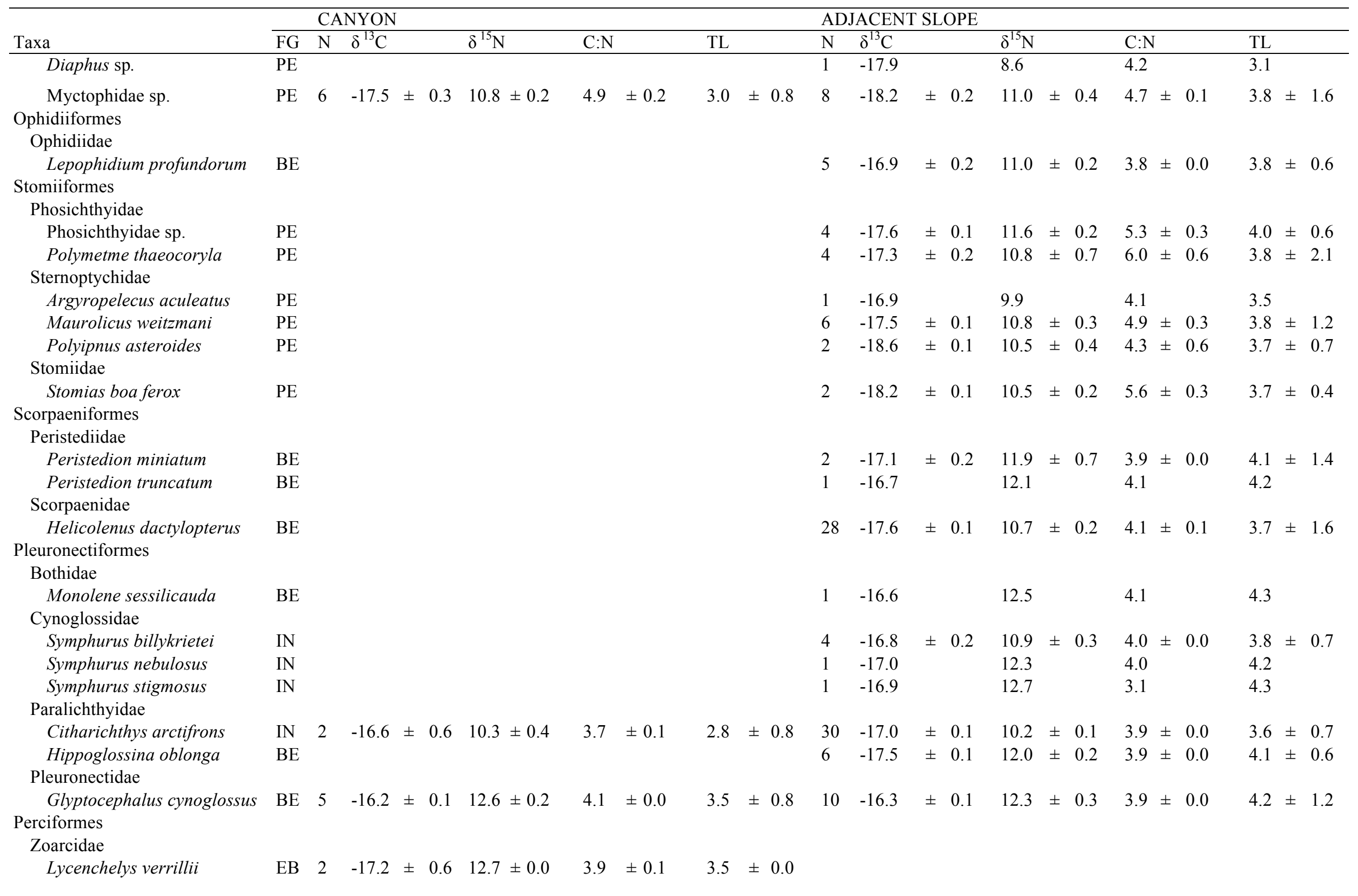




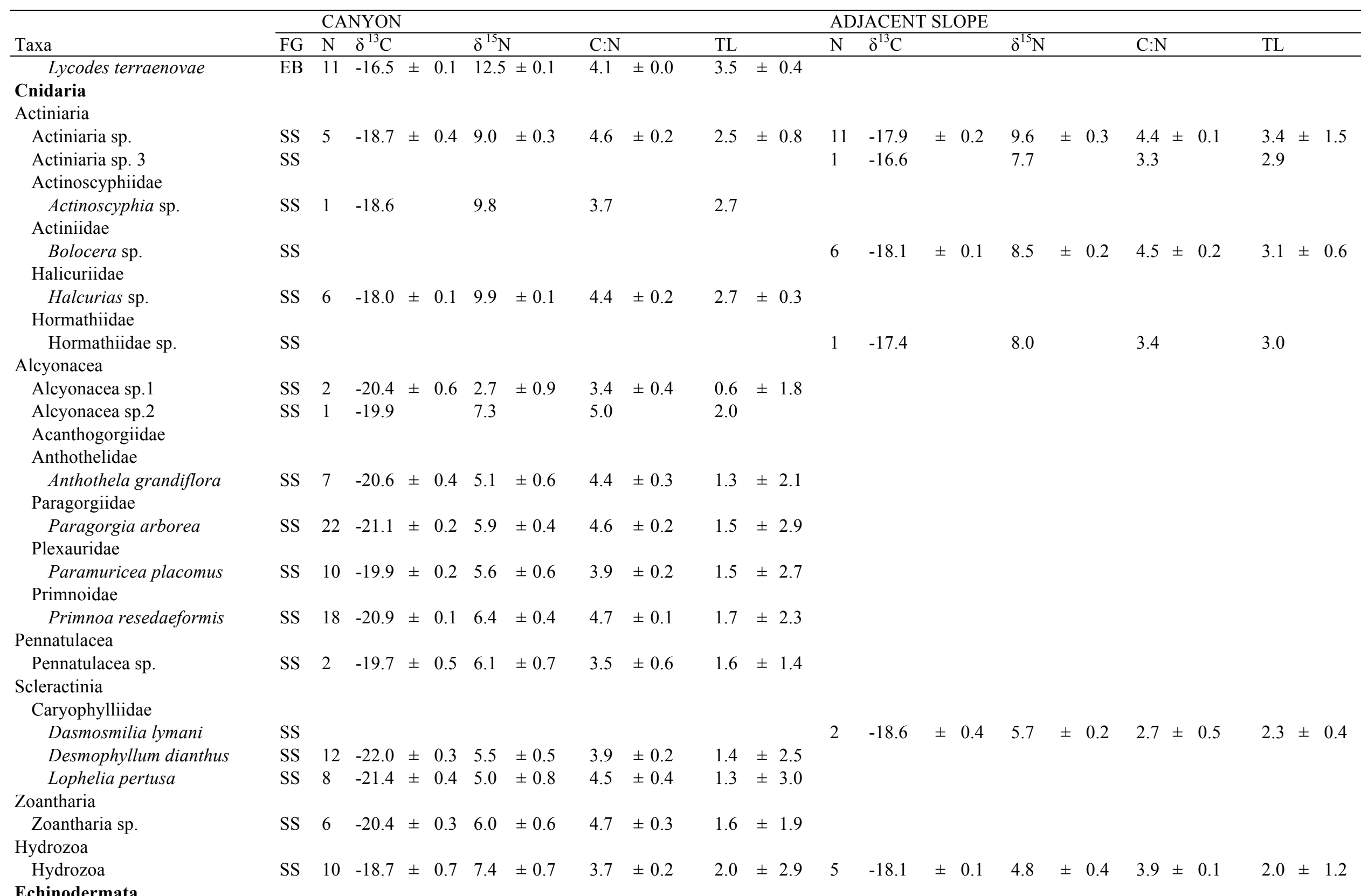




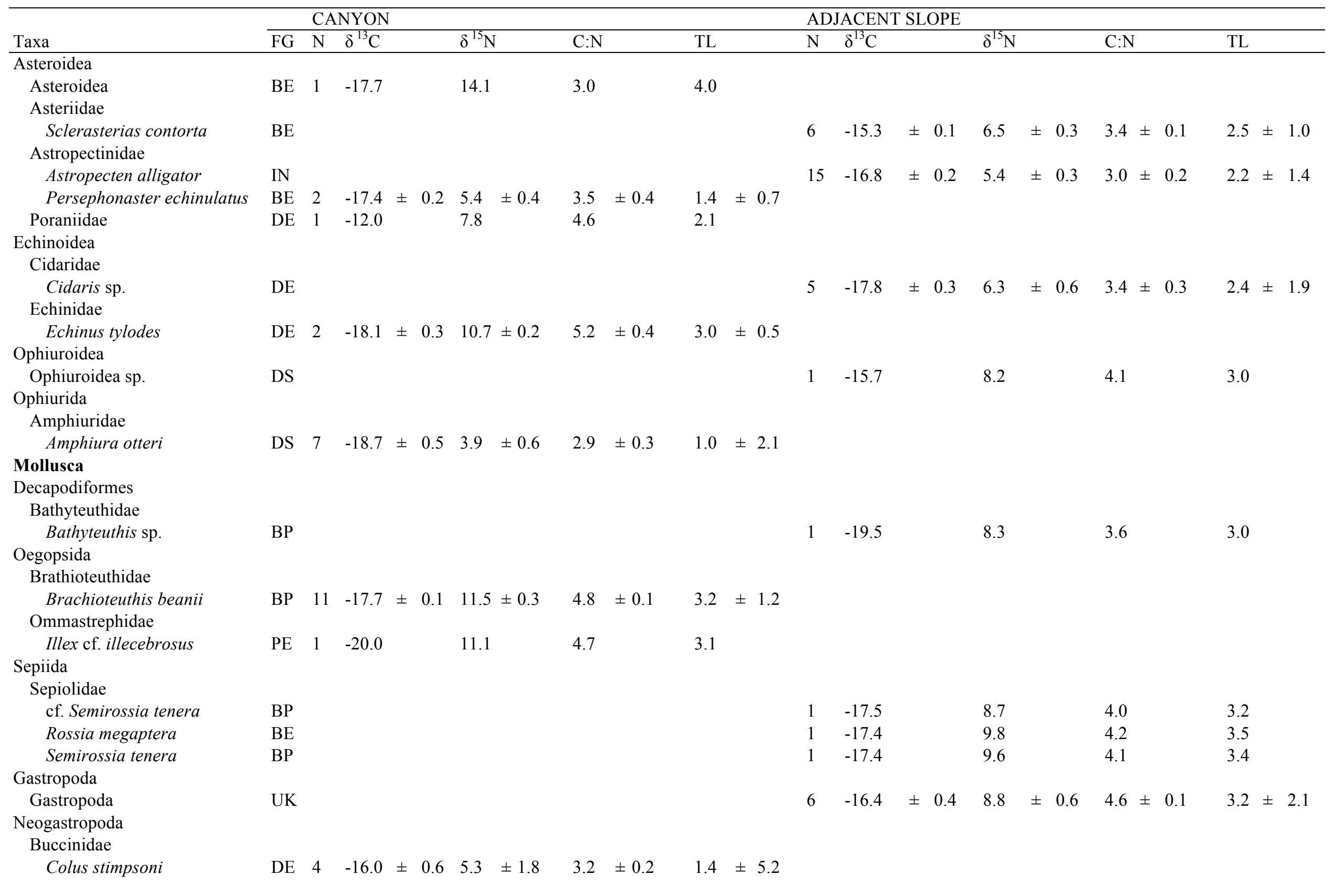




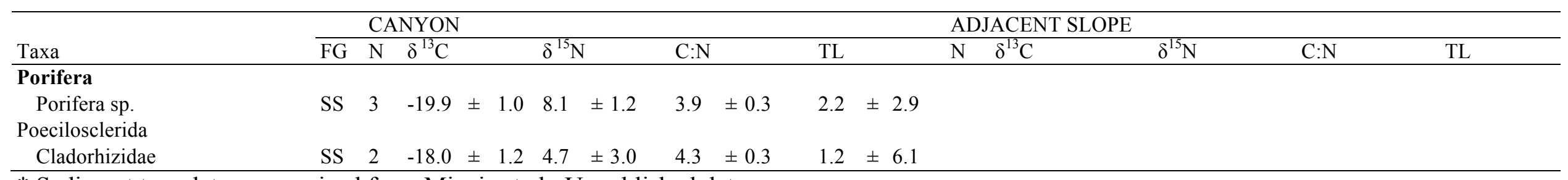

* Sediment trap data summarized from Mienis et al., Unpublished data. 
Table S4. Isotopic niche area $\left(\%^{2}\right)$ estimates (SEAc and SEAb), including $95 \%$ credible intervals (C.I.) and area overlap (\%o ${ }^{2}$ ) for different taxa groups found in Baltimore Canyon and slope environments. Bold values were significantly higher.

\begin{tabular}{|c|c|c|c|c|c|c|c|}
\hline & $\mathrm{N}$ & SEAc & SEAc overlap & SEAb & \multicolumn{3}{|c|}{ 95\% C.I. } \\
\hline \multicolumn{8}{|c|}{ Cnidaria } \\
\hline Canyon & 110.00 & 7.95 & 0.00 & 7.83 & 6.50 & to & 9.50 \\
\hline Slope & 26.00 & 3.75 & & 3.52 & 2.29 & to & 5.32 \\
\hline \multicolumn{8}{|c|}{ Echinodermata } \\
\hline Canyon & 6.00 & 31.25 & 2.88 & 22.07 & 7.76 & to & 58.55 \\
\hline Slope & 26.00 & 3.73 & & 3.49 & 2.33 & to & 5.24 \\
\hline \multicolumn{8}{|c|}{ Euphasiacea } \\
\hline Canyon & 40.00 & 2.10 & 0.33 & 2.02 & 1.47 & to & 2.76 \\
\hline Slope & 5.00 & 1.67 & & 1.14 & 0.38 & to & 3.29 \\
\hline \multicolumn{8}{|l|}{ Fish } \\
\hline Canyon & 59.00 & 2.38 & 1.74 & 2.29 & 1.78 & to & 3.00 \\
\hline Slope & 254.00 & 2.38 & & 2.37 & 2.09 & to & 2.68 \\
\hline \multicolumn{8}{|c|}{ Other Decapoda } \\
\hline Canyon & 20.00 & 2.98 & 0.42 & 2.75 & 1.74 & to & 4.35 \\
\hline Slope & 101.00 & 5.35 & & 5.32 & 4.30 & to & 6.47 \\
\hline \multicolumn{8}{|c|}{ Shrimp spp. } \\
\hline Canyon & 47.00 & 2.64 & 0.71 & 2.55 & 1.94 & to & 3.43 \\
\hline Slope & 133.00 & 3.01 & & 2.99 & 2.48 & to & 3.53 \\
\hline
\end{tabular}


Table S5. SEAc, SEAb, theta $(\theta)$ and eccentricity (E) results from $40 \%$ of data, 10 taxa, and 5 taxa.

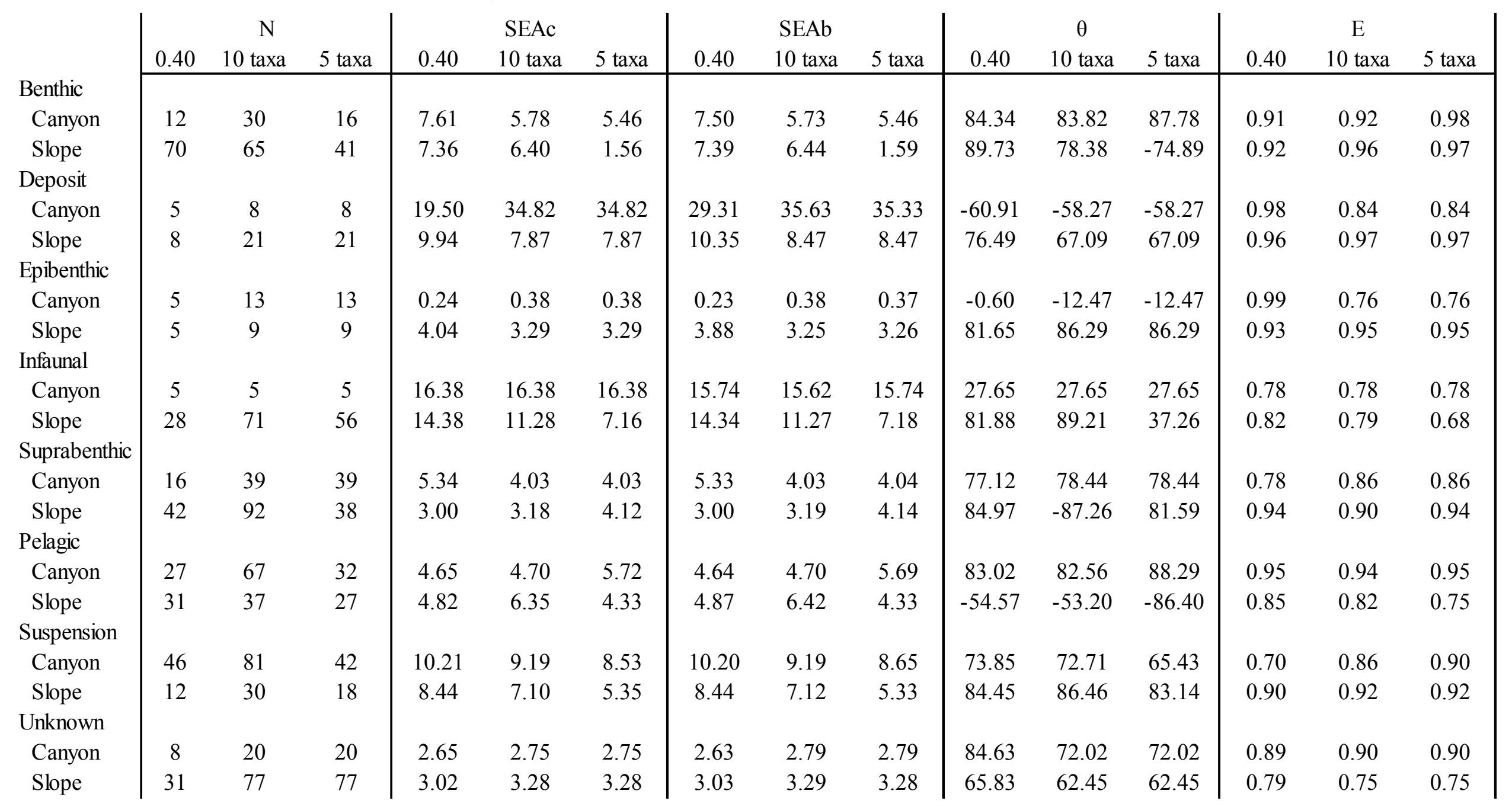


Supplementary reference list

Ammons AW, Daly M (2008) Distribution, habitat use and ecology of deepwater anemones (Actiniaria) in the Gulf of Mexico. Deep Sea Res II 55:2657-2666

Armstrong MP, Musick JA, Colvocoresses JA (1996) Food and ontogenetic shifts in feeding of the goosefish, Lophius americanus. J Northwest Atl Fish Sci 18:99-103

Auster PJ (2005) Are deep-water corals important habitats for fishes? In: Freiwald A, Roberts JM (eds) Cold-Water Corals and Ecosystems. Springer-Verlag, Berlin Heidelberg, p 747-760

Biernbaum CK, Wenner EL (1993) Trapping of necrophagous crustaceans on the upper continental slope off South Carolina, USA. J Crustac Biol 13:601-608

Blaber SJM, Bulman CM (1987) Diets of fishes of the upper continental-slope of eastern Tasmania - content, calorific values, dietary overlap and trophic relationships. Mar Biol 95:345-356

Bowman RE, Michaels WL (1984) Food of seventeen species of northwest Atlantic fish. NOAA Tech Memo, NMFS-F NEC-28

Bowman RE, Stillwell CE, Michaels WL, Grosslein MD (2000) Food of northwest Atlantic fishes and two common species of squid. NOAA Tech Memo NMFS-NE-155

Bowman TE, Gruner HE (1973) The families and genera of Hyperiidea (Crustacea:

Amphipoda). Smithsonian Contribution Zool 146:1-64

Brooks JM, Kennicutt M, Fisher CR, Macko SA, Cole K, Childress JJ, Bidigare RR, Vetter RD (1987) Deep-sea hydrocarbon seep communities:evidence for energy and nutritional carbon sources. Science 238:1138-1142

Carlier A, Guilloux EL, Olu K, Sarrazin J, Mastrototaro F, Taviani M, Clavier J (2009) Trophic relationships in a deep Mediterranean cold-water coral bank (Santa Maria di Leuca, Ionian Sea). Mar Ecol Prog Ser 397:125-137

Cartes JE (1998) Feeding strategies and partition of food resources in deep-water decapod crustaceans (400-2300 m). J Mar Biol Assoc UK 78:509-524

Cartes JE, Abelló P, Lloris D, Carbonell A, Torres P, Maynou F, Gil de Sola L (2002)

Feeding guilds of western Mediterranean demersal fish and crustaceans: an analysis based in a spring survey. Sci Mar 66:209-220

Cartes JE, Fanelli E, Papiol V, Maynou F (2010) Trophic relationships at intrannual spatial and temporal scales of macro and megafauna around a submarine canyon off the Catalonian coast (western Mediterranean). J Sea Res 63:180-190

Castro P, Williams AB, Cooper LL (2003) Revision of the family Latreilliidae Stimpson, 1858 (Crustacea, Decapoda, Brachyura). Zoosystema 25:601-634

Chintiroglou C, Koukouras A (1992) The feeding habits of three Mediterranean sea anemone species, Anemonia viridis (Forskål), Actinia equina (Linnaeus) and Cereus pedunculatus (Pennant). Helgol Meeresunters 46:53-68

Colette BB, Klein-MacPhee G (2002) Bigelow and Schroeder's fishes of the Gulf of Maine. Smithsonian Institution Press, Washington, DC

Coma R, Ribes M, Gili JM, Hughes RN (2001) The ultimate opportunists: consumers of seston. Mar Ecol Pro Ser 219:305-308

Consoli P, Battaglia P, Castriota L, Esposito V, Romeo T, Andaloro F (2010) Age, growth and feeding habits of the bluemouth rockfish, Helicolenus dactylopterus dactylopterus 
(Delaroche 1809) in the central Mediterranean (southern Tyrrhenian Sea). J Appl Ichthyol 26:583-591

Crabtree RE, Carter J, Musick JA (1991) The comparative feeding ecology of temperate and tropical deep-sea fishes from the western North-Atlantic. Deep-Sea Res I 38:1277-1298

Dalley DD, McClatchie S (1989) Functional feeding morphology of the euphausiid Nyctiphanes australis. Mar Biol 101:195-203

Dickinson JJ, Wigley RL, Brodeur RD, Brown-Leger S (1980) Distribution of gammaridean amphipoda (crustacea) in the Middle Atlantic Bight region. NOAA Tech Rep, NMFS SSRF741

Drummond-Davis NC, Mann KH, Pottle RA (1982) Some estimates of population density and feeding habits of the rock crab, Cancer irroratus, in a kelp bed in Nova Scotia. Can J Fish Aquat Sci 39:636-639

Duineveld GCA, Lavaleye MSS, Bergman MJN, de Stigter HC, Mienis F (2007) Trophic structure of a cold-water coral mound community (Rockall Bank, NE Atlantic) in relation to the near-bottom particle supply and current regime. Bull Mar Sci 81:449-467

Fanelli E, Papiol V, Cartes JE, Rumolo P, Brunet C, Sprovieri M (2011) Food web structure of the epibenthic and infaunal invertebrates on the Catalan slope (NW Mediterranean):

Evidence from ${ }^{13} \mathrm{C}$ and ${ }^{15} \mathrm{~N}$ analysis. Deep Sea Res I 58:98-109

Farlow JO. (1980) Natural history and ecology of a demersal fish-megafaunal invertebrate community from the upper continental slope off southern New England. PhD dissertation. Yale University, New Haven

Fauchald K, Jumars PA (1979) The diet of worms: a study of polychaete feeding guilds. Oceanogr Mar Biol Annu Rev 17:193-284

Freiwald A, Roberts JM (2005) Cold-water corals and ecosystems. Springer, London Gage JD, Tyler PA (1991) Deep-sea biology: a natural history of organisms at the deep-sea floor. Cambridge University Press, Cambridge

Garrison LP, Link JS (2000) Diets of five hake species in the northeast United States continental shelf ecosystem. Mar Ecol Prog Ser 204:243-255

Gerrior P (1981) The distribution and effects of fishing on the deep sea red crab, Geryon quinquedens Smith, off southern New England. MS thesis, Southeastern Massachusetts University, Dartmouth

Hopkins CCE, Sargent JR, Nilssen EM (1993) Total lipid content, and lipid and fatty acid composition of the deep-water prawn Pandalus borealis from Balsfjord, northern Norway: growth and feeding relationships. Mar Ecol Prog Ser 96:217-228

Hopkins TL, Flock ME, Gartner JV, Torres JJ (1994) Structure and trophic ecology of a low latitude midwater decapod and mysid assemblage. Mar Ecol Prog Ser 109:143-156

Horton A (2015) Feeding Habits of Demersal Fishes from Baltimore and Norfolk Canyons and Hatteras Middle Slope. MS thesis, University of North Carolina Wilmington, Wilmington, $\mathrm{NC}$

Houston KA, Haedrich RL (1986) Food habits and intestinal parasites of deep demersal fishes from the upper continental slope east of Newfoundland, northwest Atlantic Ocean. Mar Biol 92:563-574 
Hoving HJ, Perez JA, Bolstad KS, Braid HE, Evans AB, Fuchs D, Judkins H, Kelly JT, Marian JE, Nakajima R, Piatkowski U, Reid A, Vecchione M, Xavier JC (2014) The study of deep-sea cephalopods. In: Vidal EAG (ed) Advances in marine biology 67. Elsevier, Oxford

Hudson IR, Wigham BD, Billett DSM, Tyler PA (2003) Seasonality and selectivity in the feeding ecology and reproductive biology of deep-sea holothurians. Prog Oceanog 59:381407

Jangoux M, Lawrence JM (1982) Echinoderm nutrition. AA Balkema, Rotterdam

Johnson SB, Warén A, Vrijenhoek RC (2008) DNA barcoding of Lepetodrilus limpets reveals cryptic species. J Shellfish Res 27:43-51

Kapiris K (2004) Feeding ecology of Parapenaeus longirostris (Lucas, 1846) (Decapoda: Penaeidae from the Ionian Sea (central and eastern Mediterranean sea). Sci Mar 68:247-256

Keats DW, Steele DH (1990) The fourbeard rockling, Enchelyopus cimbrius (L.), in eastern Newfoundland. J Fish Biol 37:803-811

Langton RW, Bowman RE (1980) Food of fifteen northwest Atlantic gadiform fishes. NOAA Tech Rep, NMFS SSRF-740

Langton RW, Bowman RE (1981) Food of eight northwest Atlantic pleuronectiform fishes. Vol. United States Department of Commerce, NOAA Technical Report, National Marine Fisheries Service SSRF-749

Lewis JB (1982) Feeding behaviour and feeding ecology of the Octocorallia (Coelenterata: Anthozoa). J Zool 196:371-384

Link J (2002) Does food web theory work for marine ecosystems? Mar Ecol Prog Ser 230:1-9

Lovrich GA, Thiel M (2011) Ecology, physiology, feeding and trophic role of squat lobsters. In: Poore G, Ahyong S, Taylor J (eds) The biology of squat lobsters. CSIRO Publishing, Melbourne, p 183-222

MacAvoy SE, Carney RS, Fisher CR, Macko SA (2002) Use of chemosynthetic biomass by large, mobile, benthic predators in the Gulf of Mexico. Mar Ecol Prog Ser 225:65-78

Macpherson E, Roel BA (1987) Trophic relationships in the demersal fish community off Namibia. S Afr J Mar Sci 5:585-596

Madin LP (1974) Field observations on the feeding behavior of salps (Tunicata: Thaliacea). Mar Biol 25:143-147

Madurell T, Cartes JE (2006) Trophic relationships and food consumption of slope dwelling macrourids from the bathyal Ionian Sea (eastern Mediterranean). Mar Biol 148:1325-1338

Mauchline J, Fisher LR (1969) The biology of euphausiids. In: Russel FS, Yonge M. (eds) Advances in marine biology 7. Academic Press, New York

Maurer RO, Bowman RE (1975) Food habits of marine fishes of the northwest Atlantic-data report, NOAA NEFSC, Woods Hole Lab Ref Doc 75-3

McClain-Counts JP (2010) Trophic structure of mesopelagic fishes over cold seeps in the north Central Gulf of Mexico. MS thesis, University of North Carolina Wilmington, Wilmington

McEachran JD, Fechhelm JD (1998) Fishes of the Gulf of Mexico, volume 1: Myxiniformes to Gasterosteiformes,. University of Texas Press, Austin

Merrett NR, Marshall NB (1980) Observations on the ecology of deep-sea bottom-living fishes collected off northwest Africa $\left(08^{\circ}-27^{\circ} \mathrm{N}\right)$. Prog Oceanogr 9:185-244 
Mincks SL, Smith CR, Jeffreys RM, Sumida PYG (2008) Trophic structure on the West Antarctic Peninsula shelf: detritivory and benthic inertia revealed by $\delta^{13} \mathrm{C}$ and $\delta^{15} \mathrm{~N}$ analysis. Deep Sea Res II 55:2502-2514

Munroe TA (1990) Eastern Atlantic tonguefishes (Symphurus: Cynoglossidae, Pleuronectiformes), with descriptions of two new species. Bull Mar Sci 47:464-515

Neves A, Sequeira V, Paiva RB, Vieira AR, Gordo LS (2012) Feeding habits of the bluemouth, Helicolenus dactylopterus dactylopterus (Delaroche, 1809) (Pisces: Sebastidae) in the Portuguese coast. Helgol Mar Res 66:189-197

Pakhomov EA, Perissinotto R (1996) Trophodynamics of the hyperiid amphipod Themisto gaudichaudi in the South Georgia region during late austral summer. Mar Ecol Prog Ser 134:91-100

Pequegnat WE, Pequegnat LH, Kleypas JA, James BM, Kennedy EA, Hubbard GF (1983) The ecological communities of the continental slope and adjacent regimes of the northern Gulf of Mexico. Prepared for the Minerals Management Service by TerEco Corp. Contract No AA851-CT1-12:384

Powell AB, Schwartz FJ (1979) Food of Paralichthys dentatus and P. lethostigma (Pisces: Bothidae) in North Carolina estuaries. Estuaries 2:276-279

Ritz DA, Hosie GW, Kirkwood RJ (1990) Diet of Nyctiphanes australis Sars (Crustacea: Euphansiacea). Mar Freshwater Res 41:365-374

Robins CH (1968) The comparative osteology and ecology of the synaphobranchid eels of the Straits of Florida. PhD dissertation, University of Miami, Miami, FL

Rodriguez-Marin E, Punzon A, Paz J, Olaso I (1994) Feeding of most abundant fish species in Flemish Cap in summer 1993. NAFO Sci Council Res Doc 94/35, Serial No. N2403

Román E, Gonzalez C, Ceballos E (2004) Food and feeding of most abundant fish species in Flemish Cap. NAFO Sci Counceil Res Doc 04/58, Serial No. N5018

Romero MC, Lovrich GA, Tapella F, Thatje S (2004) Feeding ecology of the crab Munida subrugosa (Decapoda: Anomura: Galatheidae) in the Beagle Channel, Argentina. J Mar Biol Assoc UK 84:359-365

Sameoto DD (1980) Relationships between stomach contents and vertical migration in Meganyctiphanes norvegica, Thysanoessa raschii and T. inermis (Crustacea Euphausiacea). J Plankton Res 2:129-143

Schaffner LC, Boesch DF (1982) Spatial and temporal resource use by dominant benthic amphipoda (Ampeliscidae and Corophiidae) on the Middle Atlantic Bight outer continental shelf. Mar Ecol Prog Ser 9:231-243

Schembri PJ (1982) Feeding behaviour of fifteen species of hermit crabs (Crustacea: Decapoda: Anomura) from the Otago region, southeastern New Zealand. J Nat Hist 16:859878

Schintu P, Passariello M, Belluscio A, Ardizzone GD (1994) Growth and diet of Bothus podas (Pisces: Bothidae) in the Central Mediterranean Sea. Sci Mar 58:359-361

Scott WB, Scott MG (1988) Atlantic fishes of Canada, University of Toronto Press, Toronto

Sedberry GR, Musick JA (1978) Feeding strategies of some demersal fishes of the continental slope and rise off the mid-Atlantic coast of the USA. Mar Biol 44:357-375

Shelton RGJ (1978) On the feeding of the hagfish Myxine glutinosa in the North Sea. J Mar Biol Assoc UK 58:81-86 
Soto LA, Manickchand-Heileman S, Flores E, Licea S (2000) Processes that promote decapod diversity and abundance on the upper continental slope of the southwestern Gulf of Mexico. In: Klein JCVV, Schram FR (eds) The biodiversity crisis and crustacea, crustacean issues 12. AA Balkema, Rotterdam

Stickney RR (1976) Food habits of Georgia estuarine fishes II. Symphurus plagiusa (Pleuronectiformes: Cynoglossidae). Trans Am Fish Soc 105:202-207

Taylor JD, Taylor CN (1977) Latitudinal distribution of predatory gastropods on the eastern Atlantic shelf. J Biogeogr 4:73-81

Vacelet J, Boury-Esnault N (1995) Carnivorous sponges. Nature 373:333-335

Wildish DJ, Dadswell MJ (1985) Sublittoral gammaridean amphipods of soft sediments in the Bay of Fundy. Proc N S Inst Sci 35:1-15

Wilson SJK, Fredette TJ, Germano JD, Blake JA, Neubert PLA, Carey DA (2009) Plan-view photos, benthic grabs, and sediment-profile images: using complementary techniques to assess response to seafloor disturbance. Mar Poll Bull 59:26-37 